\title{
Global generalized solutions to a forager-exploiter model with superlinear degradation and their eventual regularity properties
}

\author{
Tobias Black*
}

\begin{abstract}
In this article we consider a cascaded taxis model for two proliferating and degrading species which thrive on the same nutrient but orient their movement according to different schemes. In particular, we assume the first group, the foragers, to orient their movement directly along an increasing gradient of the food density, while the second group, the exploiters, instead track higher densities of the forager group. Specifically, we will investigate an initial boundary-value problem for a prototypical forager-exploiter model of the form

$$
\left\{\begin{array}{lll}
u_{t}=\Delta u-\nabla \cdot(u \nabla w)+f(u), & & x \in \Omega, \quad t>0 \\
v_{t}=\Delta v-\nabla \cdot(v \nabla u)+g(v), & & x \in \Omega, \quad t>0 \\
w_{t}=\Delta w-(u+v) w-\mu w+r(x, t), & & x \in \Omega, \quad t>0
\end{array}\right.
$$

in a smoothly bounded domain $\Omega \subset \mathbb{R}^{2}$, where $\mu \geq 0, r \in C^{1}(\bar{\Omega} \times[0, \infty)) \cap L^{\infty}(\Omega \times(0, \infty))$ is nonnegative and the functions $f, g \in C^{1}([0, \infty))$ are assumed to satisfy $f(0) \geq 0, g(0) \geq 0$ as well as

$$
-k_{f} s^{\alpha}-l_{f} \leq f(s) \leq-K_{f} s^{\alpha}+L_{f} \text { and }-k_{g} s^{\beta}-l_{g} \leq g(s) \leq-K_{g} s^{\beta}+L_{g} \quad \text { for } s \geq 0,
$$

respectively, with constants $\alpha, \beta>1, k_{f}, K_{f}, k_{g}, K_{g}>0$ and $l_{f}, L_{f}, l_{g}, L_{g} \geq 0$ and $\alpha, \beta>1$.

Assuming that $\alpha>1+\sqrt{2}, \min \{\alpha, \beta\}>\frac{\alpha+1}{\alpha-1}$ and that $r$ satisfies certain structural conditions we establish the global solvability of this system with respect to a suitable generalized solution concept and then, for the more restrictive case of $\alpha, \beta>1+\sqrt{2}$ and $\mu>0$, investigate an eventual regularity effect driven by the decay of the nutrient density $w$.
\end{abstract}

Keywords: chemotaxis, social interaction, generalized solution, global existence, eventual regularity

MSC (2010): 35D99, 35B65 (primary), 35B40, 35K55, 35Q91, 35Q92, 92C17

*Institut für Mathematik, Universität Paderborn, Warburger Str. 100, 33098 Paderborn, Germany; email: tblack@math.upb.de 


\section{Introduction}

Interaction between groups of the same or even different species following distinct taxis schemes to adapt their movement is known to support rich dynamical behavior and the emergence of spatial patterns. Examples can not only be found in biological contexts of collective migration, such as swarming and flocking $([7,34,3])$, but also in other fields like economy ([10]) and criminology ([28]). A particular instance, where only two different groups are involved in the process, can be witnessed within Alaska's bird population with the macroscopic formation of shearwater flocks which orient themselves towards kittiwake foragers in order to find feeding grounds with a sufficient food source ([15]). Observations like this are the essential motivation behind the typical forager-exploiter nutrient models, in which one forager group actively searches for the food source, while the second exploiter group only indirectly finds the nutrients by tracking the actively searching foragers.

To describe a corresponding minimal model the authors of [30] proposed a cascaded taxis system essentially of the form

$$
\left\{\begin{aligned}
u_{t} & =\Delta u-\nabla \cdot(u \nabla w), \\
v_{t} & =\Delta v-\nabla \cdot(v \nabla u), \\
w_{t} & =\Delta w-(u+v) w-\mu w+r .
\end{aligned}\right.
$$

Herein, $u=u(x, t)$ denotes the time-evolution of the density of the forager population, $v=v(x, t)$ the density of the exploiter population, $w=w(x, t)$ the density of their nutrients. The degradation rate $\mu \geq 0$ is assumed to be constant and the external resupply of nutrients $r=r(x, t)$ is supposed to be a sufficiently regular nonnegative function. From an application point of view, however, it seems even more appropriate to incorporate the possibilities of degradation and proliferation for the forager and exploiter populations into the model, where, in particular, on larger time-scales for the motivating example of shearwater flocks and kittiwake foragers the death of individuals at high population densities should not be neglected. Following the approaches of related settings, this consideration would usually be integrated into the system by introducing logistic source terms of the forms $+a u-b u^{2}$ and $+a v-b v^{2}$ with $a, b>0$ to the first and second equations, respectively, where the prominent role of the exponent 2 is further underlined by its appearance in the extensively studied Fisher-KPP equation $([8,17])$. In this work, nevertheless, we are going to consider more general systems of the form

$$
\left\{\begin{array}{c}
u_{t}=\Delta u-\nabla \cdot(u \nabla w)+f(u), \\
v_{t}=\Delta v-\nabla \cdot(v \nabla u)+g(v), \\
w_{t}=\Delta w-(u+v) w-\mu w+r,
\end{array}\right.
$$

where we will assume that the prescribed kinetic source terms $f, g$ only satisfy certain growth restrictions (see (1.6) below), which ensure degradation for large population densities and thereby include spontaneous death effects.

From a mathematical point of view a cascaded taxis-mechanism of the form in (1.1) and (1.2) is quite challenging as even a single taxis term already allows for a considerable amount of mathematical questions, e.g. illustrated by the extensive studies of the acclaimed Keller-Segel chemotaxis model. (See the surveys $[4,14,23]$ for an overview of related models and results.) In order to better understand the analytical difficulty in this framework let us first take a look at the closely related setting with only one homogeneous group, where a archetypal model, often referred to as prey-taxis system $([24,43])$ or chemotaxis-consumption system $([31,32,21])$ in the literature, can be given in the form

$$
\left\{\begin{array}{c}
u_{t}=\Delta u-\chi \nabla \cdot(u \nabla w), \\
w_{t}=\Delta w-u w-\mu w+r,
\end{array}\right.
$$

where the parameter of chemotactic strength $\chi>0$ and the degradation rate $\mu \geq 0$ are constant and $r=r(x, t) \geq 0$ is sufficiently regular. Again $u=u(x, t)$ and $w=w(x, t)$ denote the density of the population and their nutrients, respectively. The prototypical case of $\mu=r=0$ has been studied rigorously and global existence and boundedness of solutions is only known under smallness conditions on the initial data $([31])$, or in a two-dimensional setting $([37,25])$, where also their stabilization towards an homogeneous equilibrium has been established for all reasonably regular initial data by utilizing the energy structure present in the system ([39]). Even in settings where additional source terms are introduced to (1.3), suitable adaptations of the energy method can provide quite extensive insight in 
the corresponding systems as e.g. witnessed by the results on global existence, boundedness and global attractors for suitably small $\chi>0$ in [43].

In the cascaded system (1.1), however, the favorable energy structure of (1.3) is missing due to the sequential attraction of the two populations and, accordingly, the analytical knowledge of the system is still quite sparse and often only covers low dimensions or generalized solution concepts. Particularly, in the one-dimensional setting the authors of [33] established global classical solutions for suitably regular initial data and, additionally, proved an exponential stabilization result under the assumption that one of the initial masses of $u$ and $v$ is sufficiently small. In higher dimensions, however, only generalized solution concepts could be considered, with [42] providing results on global generalized solutions for $n \geq 1$ whenever the initial datum for $w$ satisfies a certain smallness condition. These solutions, moreover, approach spatially homogeneous profiles in the large time limit in some topology if the supply function $r$ decays sufficiently fast. A recent study, where a volume-filling effect was included by replacing $u \nabla w$ with $u(1-u) \nabla w$ and $v \nabla u$ with $v(1-v) \nabla u$ in the first and second equation of (1.1), respectively, established global bounded classical solutions in general dimension under the assumption that the initial data for $u$ and $v$ are uniformly bounded by the density threshold 1 ([26]).

On the other hand, in single species chemotaxis-growth systems it could be observed that including a sufficiently degrading source term can have a distinct regularizing effect on the solution components. Notably, the chemotaxis systems with logistic growth terms

$$
\left\{\begin{array}{l}
u_{t}=\Delta u-\chi \nabla \cdot(u \nabla w)+a u-b u^{2}, \\
w_{t}=\Delta w-u w,
\end{array}\right.
$$

with $a \geq 0$ and $b>0$ admit global classical solutions in general dimensions if $b$ is large enough compared to the initial datum of $w$ and $\chi([21])$. In three-dimensional chemotaxis-Navier-Stokes systems the logarithmic growth term, moreover, fuels an eventual smoothing effect even when $b>0$ is arbitrary small ([20]), further highlighting the regularizing influence emitted by kinetic source terms. This is even more dominant in the classical Keller-Segel setting, where the attracting substance is produced by the population instead of consumed, i.e. replacing the second equation by $w_{t}=\Delta w-w+u$ in (1.4). This setting with $f(u) \equiv 0$ allows for blow-up solutions to exist in dimension $n=2$ if the initial mass of $u$ is large enough $([12,16,27])$ and for arbitrary mass if $n \geq 3([38])$, while all solutions are global and bounded whenever $n=2$ and $f(u)=a u-b u^{2}$ with $a \geq 0$ and $b>0$ or whenever $n \geq 3$ and $b>0$ is sufficiently large ([35]). Accordingly, source functions with a dominating death term at high population densities appear to be favorable for the global existence of solutions and we have high hopes that in the physically meaningful setting of (1.2) in a two-dimensional domain we can obtain distinctively better results beyond the quite restrictive global existence results for (1.1) mentioned above.

Main results. For the remainder of the work we are going to investigate the initial-boundary value problem

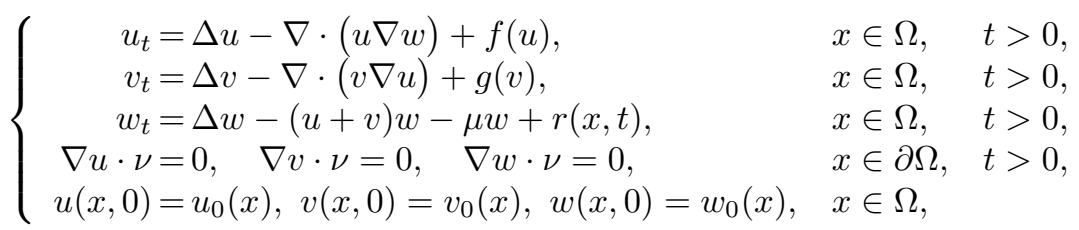

where $\nu$ denotes the outward normal vector field on $\partial \Omega$ and $f, g \in C^{1}([0, \infty))$ satisfy

$$
\begin{gathered}
-k_{f} s^{\alpha}-l_{f} \leq f(s) \leq-K_{f} s^{\alpha}+L_{f} \text { and }-k_{g} s^{\beta}-l_{g} \leq g(s) \leq-K_{g} s^{\beta}+L_{g}, \quad \text { for } s \geq 0 \\
\text { as well as } f(0) \geq 0, \quad g(0) \geq 0,
\end{gathered}
$$

with constants $K_{f}, K_{g}, k_{f}, k_{g}>0, L_{f}, L_{g}, l_{f}, l_{g} \geq 0$ and $\alpha, \beta>1$. As for the other ingredients of (1.5) we merely require that $\mu$ is a nonnegative constant, that $r \in C^{1}(\bar{\Omega} \times[0, \infty)) \cap L^{\infty}(\Omega \times(0, \infty))$ is nonnegative with

$$
r_{*}:=\sup _{s \in(0, \infty)}\|r(\cdot, s)\|_{L^{\infty}(\Omega)}<\infty
$$

and that the initial data $\left(u_{0}, v_{0}, w_{0}\right)$ satisfy the conditions

$$
\left\{\begin{array}{c}
u_{0} \in W^{1, \infty}(\Omega) \text { is nonnegative with } u_{0} \not \equiv 0 \\
v_{0} \in W^{1, \infty}(\Omega) \text { is nonnegative with } v_{0} \not \equiv 0 \\
w_{0} \in W^{2, \infty}(\Omega) \text { is nonnegative with } \nabla w_{0} \cdot \nu=0 \text { on } \partial \Omega .
\end{array}\right.
$$


In this setting our first result is concerned with the global existence of generalized solutions for kinetic functions $f$ and $g$ satisfying (1.6) with suitably large degradation rates $\alpha, \beta>1$ and reads as follows.

Theorem 1.1.

Let $\Omega \subset \mathbb{R}^{2}$ be a bounded domain with smooth boundary. Suppose that $\mu \geq 0$, that the functions $f$ and $g$ fulfill (1.6) with $K_{f}, K_{g}, k_{f}, k_{g}>0, L_{f}, L_{g}, l_{f}, l_{g} \geq 0, \alpha>1+\sqrt{2}$ and $\beta>1$ satisfying

$$
\min \{\alpha, \beta\}>\frac{\alpha+1}{\alpha-1}
$$

and that $r \in C^{1}(\bar{\Omega} \times[0, \infty)) \cap L^{\infty}(\Omega \times(0, \infty))$ is nonnegative and fulfills (1.7). Then, for any $u_{0}, v_{0}$ and $w_{0}$ complying with (1.8), the system (1.5) admits at least one global generalized solution $(u, v, w)$ in the sense of Definition 2.1.

The prominent degradation exponent 2, which is commonly found in studies of systems with only a single taxis term, is not covered by the result above and hence the question whether in the cascaded taxis system logistic growth terms are strong enough to ensure global solutions in quite mild solution concepts has to be left for further research. Nevertheless, physically meaningful source terms, e.g. functions describing an Allee-effect, are still contained in the theorem above. We remark the following.

\section{Remark 1.2.}

i) We note that if $\alpha \leq \beta$, condition (1.9) is always satisfied due to $\alpha>1+\sqrt{2}$, whereas for large $\alpha>\beta$ the fraction $\frac{\bar{\alpha}+1}{\alpha-1}$ tends to 1 and hence if $\alpha$ is suitably large, the degradation function $g$ may also be of a subquadratic but superlinear form.

ii) Kinetic source terms like $f(s) \equiv s(1-s)(s-2)$, which are prototypical choices when describing a population evolution obeying certain Allee-effects, satisfy the conditions of Theorem 1.1.

In our second result we investigate a slightly more restrictive setting, where we assume that in addition to all the requirements of Theorem 1.1 we also have $\beta>1+\sqrt{2}, \mu>0$ and that $r \in C^{1}(\bar{\Omega} \times[0, \infty)) \cap$ $L^{\infty}(\Omega \times(0, \infty)) \cap L^{1}\left((0, \infty) ; L^{\infty}(\Omega)\right)$ satisfies

$$
r_{* *}:=\int_{0}^{\infty}\|r(\cdot, t)\|_{L^{\infty}(\Omega)} \mathrm{d} t<\infty .
$$

These conditions allow for an eventual regularization process to occur, which entails that after some (possibly large) waiting time $T_{\diamond}>0$ the destabilizing effect of the cascaded taxis terms is mitigated by the sufficiently strong degradation of the forager and exploiter groups and the suitably fast decaying resupply of nutrients implied by (1.10), so that the solution to (1.5) actually becomes a classical solution after some time.

\section{Theorem 1.3.}

Let $\Omega \subset \mathbb{R}^{2}$ be a bounded domain with smooth boundary. Suppose that $\mu>0$, that the functions $f$ and $g$ fulfill (1.6) with $K_{f}, K_{g}, k_{f}, k_{g}>0, L_{f}, L_{g}, l_{f}, l_{g} \geq 0, \alpha>1+\sqrt{2}$ and $\beta>1+\sqrt{2}$ and that $r \in C^{1}(\bar{\Omega} \times[0, \infty)) \cap L^{\infty}(\Omega \times(0, \infty)) \cap L^{1}\left((0, \infty) ; L^{\infty}(\Omega)\right)$ is nonnegative and fulfills $(1.7)$ and $(1.10)$. Then there exists $T_{\diamond}>0$ such that the global generalized solution of (1.5) from Theorem 1.1 has the properties

$$
u \in C^{2,1}\left(\bar{\Omega} \times\left[T_{\diamond}, \infty\right)\right), \quad v \in C^{2,1}\left(\bar{\Omega} \times\left[T_{\diamond}, \infty\right)\right), \quad \text { and } \quad w \in C^{2,1}\left(\bar{\Omega} \times\left[T_{\diamond}, \infty\right)\right),
$$

and such that $(u, v, w)$ solves (1.5) classically in $\Omega \times\left(T_{\diamond}, \infty\right)$.

Outline of the approach. After a brief introduction of the generalized solution concept we are going to consider (Definition 2.1), we will propose a family of regularized initial boundary-value problems in Section 3 and, starting with a time-local existence result, provide uniform $L^{1}$ bounds for both $u_{\varepsilon}$ and $v_{\varepsilon}$ and a uniform $L^{\infty}$ bound on $w_{\varepsilon}$, which, when combined with semigroup arguments, entail the time-global existence of the approximating system for any fixed $\varepsilon \in(0,1)$ (Lemma 3.4). Section 4 is concerned with the derivation of essential a priori estimates, which constitute the main part of the proof of Theorem 1.1. In particular, we will draw on the well-known maximal Sobolev regularity estimates for the Neumann heat semigroup employed to the third equation (Lemma 4.3) to obtain sufficient control on the terms appearing during the testing procedure for $u_{\varepsilon}$ (Lemma 4.6). The second quantity will be 
treated by considering the time-evolution of $\int_{\Omega} \ln \left(v_{\varepsilon}+1\right)$ (Lemma 4.8), which offers very mild, yet still sufficient, information on the spatial gradient of $v_{\varepsilon}$. In Section 5 we prepare additional a priori estimates necessary for the limiting procedure undertaken in Proposition 6.1 of Section 6. In Section 7 we will then conclude the proof of Theorem 1.1 by verifying that the obtained limit object from Section 6 is in fact a global generalized solution.

The proof of Theorem 1.3 will be in the focus of Sections 8-11, with Section 8 being dedicated to conditional regularity estimates which provide the basis for the bootstrapping procedure undertaken in Section 11. Sections 9 and 10 ensure that the conditions required by the conditional estimates of Lemma 8.2 are indeed fulfilled. Specifically, we make use of (1.10) and the fact that $\mu>0$ to prove the decay of $w_{\varepsilon}$ in the $L^{\infty}$ norm in Lemma 9.2 and then investigate $\frac{\mathrm{d}}{\mathrm{d} t} \int_{\Omega} \frac{u_{\varepsilon}^{q}}{\left(2 \delta-w_{\varepsilon}\right)^{\theta}}$ for suitably small $\delta>0$ and $\theta>0$ to establish an eventual $L^{q}$ bound for $u_{\varepsilon}$ in Lemma 10.2. Section 11 finalizes the proof of Theorem 1.3 by the aforementioned bootstrapping procedure based on Lemma 8.2.

\section{Global solutions in a generalized solution concept}

Since both of our theorems are concerned with global generalized solutions, let us first specify what exactly constitutes a solution in this notion. The main difference to the standard concept of weak solvability consists of only requiring a integral inequality corresponding to the variational interpretation of the second equation of (1.5). This in turn allows us to prescribe milder regularity assumptions for the second solution component, which certainly is the hardest to obtain for the system in question. Solution concepts of this form have been successfully employed in many chemotaxis contexts, see e.g. [40, 22].

\section{Definition 2.1.}

A triple $(u, v, w)$ of functions

$$
u \in L_{l o c}^{2}\left([0, \infty) ; W^{1,2}(\Omega)\right), \quad v \in L_{l o c}^{1}(\bar{\Omega} \times[0, \infty)), \quad w \in L_{l o c}^{\infty}(\bar{\Omega} \times[0, \infty)) \cap L_{l o c}^{2}\left([0, \infty) ; W^{1,2}(\Omega)\right)
$$

such that

$$
f(u) \quad \text { and } \quad \frac{g(v)}{v+1} \quad \text { belong to } L_{l o c}^{1}(\bar{\Omega} \times[0, \infty)) \quad \text { and that } \quad \nabla \ln (v+1) \in L_{l o c}^{2}\left(\bar{\Omega} \times[0, \infty) ; \mathbb{R}^{2}\right),
$$

and such that $u \geq 0, v \geq 0$ and $w \geq 0$ in $\bar{\Omega} \times[0, \infty)$ will be called a global generalized solution of (1.5) if the inequality

$$
\begin{gathered}
-\int_{0}^{\infty} \int_{\Omega} \ln (v+1) \psi_{t}-\int_{\Omega} \ln \left(v_{0}+1\right) \psi(\cdot, 0) \\
\geq \int_{0}^{\infty} \int_{\Omega}|\nabla \ln (v+1)|^{2} \psi-\int_{0}^{\infty} \int_{\Omega} \nabla \ln (v+1) \cdot \nabla \psi-\int_{0}^{\infty} \int_{\Omega} \frac{v}{v+1}(\nabla \ln (v+1) \cdot \nabla u) \psi \\
+\int_{0}^{\infty} \int_{\Omega} \frac{v}{v+1} \nabla u \cdot \nabla \psi+\int_{0}^{\infty} \int_{\Omega} \frac{g(v)}{v+1} \psi
\end{gathered}
$$

holds for every nonnegative $\psi \in C_{0}^{\infty}(\bar{\Omega} \times[0, \infty))$, if the identities

$$
-\int_{0}^{\infty} \int_{\Omega} u \varphi_{t}-\int_{\Omega} u_{0} \varphi(\cdot, 0)=-\int_{0}^{\infty} \int_{\Omega} \nabla u \cdot \nabla \varphi+\int_{0}^{\infty} \int_{\Omega} u \nabla w \cdot \nabla \varphi+\int_{0}^{\infty} \int_{\Omega} f(u) \varphi
$$

and

$$
-\int_{0}^{\infty} \int_{\Omega} w \varphi_{t}-\int_{\Omega} w_{0} \varphi(\cdot, 0)=-\int_{0}^{\infty} \int_{\Omega} \nabla w \cdot \nabla \varphi-\int_{0}^{\infty} \int_{\Omega}(u+v) w \varphi-\mu \int_{0}^{\infty} \int_{\Omega} w \varphi+\int_{0}^{\infty} \int_{\Omega} r \varphi
$$

are fulfilled for every $\varphi \in C_{0}^{\infty}(\bar{\Omega} \times[0, \infty))$ and if finally the inequality

$$
\int_{\Omega} v(\cdot, t) \leq \int_{\Omega} v_{0}+\int_{0}^{t} \int_{\Omega} g(v)
$$

holds for a.e. $t \in(0, \infty)$. 
We note that an estimate of the form (2.4) is a necessary requirement, if we want to have a concept of generalized solvability, which is consistent with the concept of classical solvability in the following sense.

Remark 2.2. Whenever a global generalized solution $(u, v, w)$ satisfies $u, v, w \in C^{0}(\bar{\Omega} \times[0, \infty)) \cap$ $C^{2,1}(\bar{\Omega} \times(0, \infty))$, it can easily be checked that the global generalized solution is also a global classical solution of (1.5) in $\Omega \times(0, \infty)$. (See [40, Lemma 2.1] and [22, Lemma 2.5] for the essential arguments involved.)

\section{Global solutions to approximating systems and basic properties}

Global generalized solutions in the sense of the Definition 2.1 above will be obtained as limit of global classical solutions to the following family of regularized systems

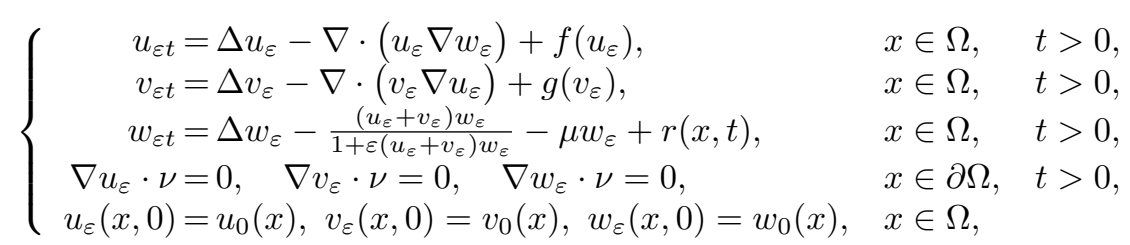

where in particular the fact that $\frac{\xi}{1+\varepsilon \xi} \leq \frac{1}{\varepsilon}$ for all $\varepsilon \in(0,1)$ and $\xi \geq 0$ enables us to obtain quite strong regularity estimates on the third solution component for each fixed $\varepsilon \in(0,1)$, providing an important tool when trying to show that for each $\varepsilon \in(0,1)$ the solution to $(3.1)$ is global in time. As we still need a few additional ingredients, however, let us begin our analysis of this family of approximating systems with merely establishing time-local existence of classical solutions to (3.1).

\section{Lemma 3.1.}

Let $\Omega \subset \mathbb{R}^{2}$ be a bounded domain with smooth boundary. Suppose that the functions $f$ and $g$ fulfill (1.6) with $K_{f}, K_{g}, k_{f}, k_{g}>0, L_{f}, L_{g}, l_{f}, l_{g} \geq 0$ and some $\alpha, \beta>1$. Assume $\mu \geq 0$ and that $r \in C^{1}(\bar{\Omega} \times[0, \infty)) \cap L^{\infty}(\Omega \times(0, \infty))$ is nonnegative and satisfies $(1.7)$. Then, for any $u_{0}, v_{0}$ and $w_{0}$ complying with $(1.8)$ and any $\varepsilon \in(0,1)$ there exist $T_{\max , \varepsilon}>0$ and functions

$$
\left\{\begin{array}{l}
u_{\varepsilon} \in \bigcap_{q>n} C^{0}\left(\left[0, T_{\max , \varepsilon}\right) ; W^{1, q}(\Omega)\right) \cap C^{2,1}\left(\bar{\Omega} \times\left[0, T_{\max , \varepsilon}\right)\right), \\
v_{\varepsilon} \in \bigcap_{q>n} C^{0}\left(\left[0, T_{\max , \varepsilon}\right) ; W^{1, q}(\Omega)\right) \cap C^{2,1}\left(\bar{\Omega} \times\left[0, T_{\max , \varepsilon}\right)\right), \\
w_{\varepsilon} \in \bigcap_{q>n} C^{0}\left(\left[0, T_{\max , \varepsilon}\right) ; W^{1, q}(\Omega)\right) \cap C^{2,1}\left(\bar{\Omega} \times\left[0, T_{\max , \varepsilon}\right)\right),
\end{array}\right.
$$

which solve $(1.5)$ in $\Omega \times\left(0, T_{\max , \varepsilon}\right)$ in the classical sense and satisfy $u_{\varepsilon} \geq 0, v_{\varepsilon} \geq 0$ and $w_{\varepsilon} \geq 0$ in $\bar{\Omega} \times(0, \infty)$. Moreover, either $T_{\max , \varepsilon}=\infty$ or

$$
\limsup _{t \nearrow T_{\max , \varepsilon}}\left(\left\|u_{\varepsilon}(\cdot, t)\right\|_{W^{1, p}(\Omega)}+\left\|v_{\varepsilon}(\cdot, t)\right\|_{W^{1, p}(\Omega)}+\left\|w_{\varepsilon}(\cdot, t)\right\|_{W^{1, p}(\Omega)}\right)=\infty
$$

for all $p>2$.

Proof: The time-local existence of a classical solution $\left(u_{\varepsilon}, v_{\varepsilon}, w_{\varepsilon}\right)$ satisfying (3.2) is a consequence of Amann's general theory on quasilinear parabolic boundary value problems (cf. [1, Theorem 14.5 and Theorem 14.6]) with the extensibility criterion (3.3) entailed by [1, Theorem 15.5]. The nonnegativity of the solution components is then ensured by employing the parabolic comparison principle to the subsolution 0 .

For the remainder of this work we will always assume that all conditions of Lemma 3.1 are satisfied and for $\varepsilon \in(0,1)$ we will always denote by $\left(u_{\varepsilon}, v_{\varepsilon}, w_{\varepsilon}\right)$ the solution provided by Lemma 3.1 and with $T_{\max , \varepsilon} \in(0, \infty]$ its corresponding maximal existence time.

Relying on our standing assumption (1.6), we can integrate the first and second equations of (3.1) to obtain an $L^{1}$ bound uniform in $\varepsilon \in(0,1)$ for both $u_{\varepsilon}$ and $v_{\varepsilon}$.

Lemma 3.2.

For any $\varepsilon \in(0,1)$ and all $t \in\left(0, T_{\max , \varepsilon}\right)$ the solution $\left(u_{\varepsilon}, v_{\varepsilon}, w_{\varepsilon}\right)$ of $(3.1)$ obtained in Lemma 3.1 
satisfies

$$
\int_{\Omega} u_{\varepsilon}(\cdot, t) \leq \max \left\{\int_{\Omega} u_{0},|\Omega|\left(\frac{L_{f}}{K_{f}}\right)^{\frac{1}{\alpha}}\right\}=: u_{*} \quad \text { and } \quad \int_{\Omega} v_{\varepsilon}(\cdot, t) \leq \max \left\{\int_{\Omega} v_{0},|\Omega|\left(\frac{L_{g}}{K_{g}}\right)^{\frac{1}{\beta}}\right\}=: v_{*} .
$$

Proof: Making use of the first equation and the prescribed boundary conditions of (3.1), we can draw on integration by parts and (1.6) to find that

$$
\frac{\mathrm{d}}{\mathrm{d} t} \int_{\Omega} u_{\varepsilon}=\int_{\Omega} f\left(u_{\varepsilon}\right) \leq-K_{f} \int_{\Omega} u_{\varepsilon}^{\alpha}+L_{f}|\Omega|
$$

holds on $\left(0, T_{\max , \varepsilon}\right)$. Due to $\alpha>1$ an application of Hölder's inequality entails that $y_{\varepsilon}(t):=\int_{\Omega} u_{\varepsilon}(\cdot, t)$ satisfies the differential inequality

$$
y_{\varepsilon}^{\prime}(t)+\frac{K_{f}}{|\Omega|^{\alpha-1}} y_{\varepsilon}^{\alpha}(t) \leq L_{f}|\Omega| \quad \text { for all } t \in\left(0, T_{\max , \varepsilon}\right),
$$

An ODE comparison argument with $u_{*}$ then yields the asserted $L^{1}$ bound for $u_{\varepsilon}$. Repeating similar arguments for the second equation of (3.1) we also conclude the desired bound for $\int_{\Omega} v_{\varepsilon}$.

Emulating arguments applied to the system without kinetic source functions, we can make use of the same supersolution as in [42, Lemma 3.1] to easily conclude the uniform $L^{\infty}$ boundedness of $w_{\varepsilon}$ by a straightforward comparison argument.

\section{Lemma 3.3.}

For any $\varepsilon \in(0,1)$ and all $t \in\left(0, T_{\max , \varepsilon}\right)$ the solution $\left(u_{\varepsilon}, v_{\varepsilon}, w_{\varepsilon}\right)$ of $(3.1)$ satisfies

$$
\left\|w_{\varepsilon}(\cdot, t)\right\|_{L^{\infty}(\Omega)} \leq\left\|w_{0}\right\|_{L^{\infty}(\Omega)}+\frac{r_{*}}{\mu}=: w_{*} .
$$

Proof: For $x \in \bar{\Omega}$ and $t \in(0, \infty)$ we let

$$
\bar{w}(x, t):=\left\|w_{0}\right\|_{L^{\infty}(\Omega)} e^{-\mu t}+\int_{0}^{t} e^{-\mu(t-s)}\|r(\cdot, s)\|_{L^{\infty}(\Omega)} \mathrm{d} s .
$$

Then, we observe that $\bar{w}$ is spatially homogeneous for each $t \in\left(0, T_{\max , \varepsilon}\right)$ with $\bar{w} \leq w_{*}$ on $\bar{\Omega} \times\left(0, T_{\max , \varepsilon}\right)$ and satisfies

$$
\bar{w}_{t}-\Delta \bar{w}+\frac{\left(u_{\varepsilon}+v_{\varepsilon}\right) w_{\varepsilon}}{1+\varepsilon\left(u_{\varepsilon}+v_{\varepsilon}\right) w_{\varepsilon}}+\mu \bar{w}-r(x, t) \geq \bar{w}_{t}+\mu \bar{w}-\|r(\cdot, t)\|_{L^{\infty}(\Omega)}=0 \quad \text { on } \Omega \times\left(0, T_{\max , \varepsilon}\right),
$$

due to the nonnegativity of $u_{\varepsilon}, v_{\varepsilon}$ and $w_{\varepsilon}$. Hence, an application of the comparison principle entails that $\bar{w} \geq w_{\varepsilon}$ on $\Omega \times\left(0, T_{\max , \varepsilon}\right)$ and, in particular, $\left\|w_{\varepsilon}(\cdot, t)\right\|_{L^{\infty}(\Omega)} \leq w_{*}$ for all $t \in\left(0, T_{\max , \varepsilon}\right)$.

The $L^{\infty}$ information for $w_{\varepsilon}$ at hand we can now extract sufficient information to verify that for any fixed $\varepsilon \in(0,1)$ the solution is global in time.

\section{Lemma 3.4.}

For any $\varepsilon \in(0,1)$ the solution $\left(u_{\varepsilon}, v_{\varepsilon}, w_{\varepsilon}\right)$ of $(3.1)$ obtained in Lemma 3.1 is global, i.e. $T_{\max , \varepsilon}=\infty$.

Proof: We augment the arguments presented in [42, Lemma 2.1] to cover the present setting. We fix a suitably large $p>2$ and assume that $T_{\max , \varepsilon}<\infty$. Due to $\frac{\xi}{1+\varepsilon \xi} \leq \frac{1}{\varepsilon}$ for all $\xi \geq 0$ and $r \leq r_{*}$ in $\Omega \times\left(0, T_{\max , \varepsilon}\right)$ we can on the one hand utilize well-known maximal Sobolev regularity theory (see e.g. $[13,11])$, Lemma 3.3 and (1.8) to obtain $C_{1}>0$ such that

$$
\begin{aligned}
& \int_{0}^{T_{\max , \varepsilon}}\left\|w_{\varepsilon t}\right\|_{L^{p}(\Omega)}^{p}+\int_{0}^{T_{\max , \varepsilon}}\left\|w_{\varepsilon}\right\|_{W^{2, p}(\Omega)}^{p} \\
\leq & C_{1}\left\|w_{0}\right\|_{W^{2, p}(\Omega)}^{p}+C_{1} \int_{0}^{T_{\max , \varepsilon}}\left(\left\|\frac{\left(u_{\varepsilon}+v_{\varepsilon}\right) w_{\varepsilon}}{1+\varepsilon\left(u_{\varepsilon}+v_{\varepsilon}\right) w_{\varepsilon}}\right\|_{L^{p}(\Omega)}^{p}+\left\|\mu w_{\varepsilon}\right\|_{L^{p}(\Omega)}^{p}+\|r\|_{L^{p}(\Omega)}^{p}\right) \\
\leq & C_{1}\left(\left\|w_{0}\right\|_{W^{2, p}(\Omega)}^{p}+\varepsilon^{-p}|\Omega|+\mu^{p} w_{*}^{p}|\Omega|+r_{*}^{p}|\Omega|\right) T_{\max , \varepsilon},
\end{aligned}
$$


and on the other hand employ semigroup techniques (e.g. [36, Lemma 1.3]) to obtain $C_{2}>0$ such that also $\left\|\nabla w_{\varepsilon}\right\|_{L^{\infty}(\Omega)} \leq C_{2}$ holds on $\left(0, T_{\max , \varepsilon}\right)$. This information at hand, we perform testing procedures on the first equation of (3.1) and rely on the fact that $\alpha>1$ to obtain $C_{3}>0$ such that that $\left\|u_{\varepsilon}\right\|_{L^{4}(\Omega)} \leq C_{3}$ on $\left(0, T_{\max , \varepsilon}\right)$, which, by utilizing the semigroup estimates for the Neumann heat semigroup once more, can also be refined into a bound for $\left\|u_{\varepsilon}\right\|_{L^{\infty}(\Omega)}$ on $\left(0, T_{\max , \varepsilon}\right)$. In light of the properties assumed for $f$, the boundedness of $u_{\varepsilon}$ also readily entails the existence of $C_{4}>0$ such that $\left\|f\left(u_{\varepsilon}\right)\right\|_{L^{\infty}(\Omega)} \leq C_{4}$ on $\left(0, T_{\max , \varepsilon}\right)$. Hence, we may interpret $u_{\varepsilon}$ as a weak solution of the equation

$$
\tilde{u}_{t}=\Delta \tilde{u}+a(x, t) \cdot \nabla \tilde{u}+b(x, t) \tilde{u}+c(x, t) \quad \text { on } \Omega \times\left(0, T_{\max , \varepsilon}\right),
$$

with $a(x, t):=-\nabla w_{\varepsilon} \in L^{\infty}\left(\Omega \times\left(0, T_{\max , \varepsilon}\right) ; \mathbb{R}^{2}\right), b(x, t)=-\Delta w_{\varepsilon} \in L^{p}\left(\Omega \times\left(0, T_{\max , \varepsilon}\right)\right)$ and $c(x, t):=$ $f\left(u_{\varepsilon}\right) \in L^{\infty}\left(\Omega \times\left(0, T_{\max , \varepsilon}\right)\right)$, where we can now draw on standard theory to conclude that also $u_{\varepsilon} \in L^{\infty}\left(\left(0, T_{\max , \varepsilon}\right) ; W^{1, \infty}(\Omega)\right) \cap L^{p}\left(\left(0, T_{\max , \varepsilon}\right) ; W^{2, p}(\Omega)\right)$. With this we may repeat the steps employed to $u_{\varepsilon}$ again for the second equation of (3.1) to obtain $v_{\varepsilon} \in L^{\infty}\left(\left(0, T_{\max , \varepsilon}\right) ; W^{1, \infty}(\Omega)\right) \cap$ $L^{p}\left(\left(0, T_{\max , \varepsilon}\right) ; W^{2, p}(\Omega)\right)$, which finally contradicts the extensibility criterion (3.3) and hence our assumption $T_{\max , \varepsilon}<\infty$ has to be false.

\section{Essential uniform estimates}

This section is devoted to the establishment of estimates which build the essential groundwork for later refinement in Section 5 and the limit procedure in Section 6. This section will also feature the main reasons behind the constraints imposed on the parameters $\alpha$ and $\beta$ in Theorem 1.1.

We start by noting that an integration of the first and second equations of (3.1) also entails some additional information we did not mention in Lemma 3.2. In particular, we also obtain an inequality for $v_{\varepsilon}$ corresponding to (2.4) of the generalized solution concept.

\section{Lemma 4.1.}

Let $u_{*}$ and $v_{*}$ be provided by (3.4). For any $\varepsilon \in(0,1)$ the solution $\left(u_{\varepsilon}, v_{\varepsilon}, w_{\varepsilon}\right)$ of $(3.1)$ fulfills

$$
\int_{t}^{t+1} \int_{\Omega} u_{\varepsilon}^{\alpha} \leq \frac{L_{f}|\Omega|}{K_{f}}+\frac{u_{*}}{K_{f}} \quad \text { and } \quad \int_{t}^{t+1} \int_{\Omega} v_{\varepsilon}^{\beta} \leq \frac{L_{g}|\Omega|}{K_{g}}+\frac{v_{*}}{K_{g}}
$$

for all $t>0$. Moreover, for any $\varepsilon \in(0,1)$ and all $t>0$ the solution satisfies

$$
\int_{\Omega} v_{\varepsilon}(\cdot, t)=\int_{\Omega} v_{0}+\int_{0}^{t} \int_{\Omega} g\left(v_{\varepsilon}\right) .
$$

Proof: Again by integrating the first and second equations of (3.1) over $\Omega$ we first find that

$$
\frac{\mathrm{d}}{\mathrm{d} t} \int_{\Omega} u_{\varepsilon}=\int_{\Omega} f\left(u_{\varepsilon}\right) \text { and } \frac{\mathrm{d}}{\mathrm{d} t} \int_{\Omega} v_{\varepsilon}=\int_{\Omega} g\left(v_{\varepsilon}\right) \text { hold on }(0, \infty) .
$$

Plugging in (1.6) we obtain that

$$
\frac{\mathrm{d}}{\mathrm{d} t} \int_{\Omega} u_{\varepsilon}+K_{f} \int_{\Omega} u_{\varepsilon}^{\alpha} \leq L_{f}|\Omega| \quad \text { and } \quad \frac{\mathrm{d}}{\mathrm{d} t} \int_{\Omega} v_{\varepsilon}+K_{g} \int_{\Omega} v_{\varepsilon}^{\beta}=L_{g}|\Omega|
$$

are valid on $(0, \infty)$. Since $u_{\varepsilon}$ and $v_{\varepsilon}$ are nonnegative, we conclude upon integration from $t$ to $t+1$ that

$$
K_{f} \int_{t}^{t+1} \int_{\Omega} u_{\varepsilon}^{\alpha} \leq \int_{\Omega} u_{\varepsilon}(\cdot, t)+L_{f}|\Omega| \quad \text { and } \quad K_{g} \int_{t}^{t+1} \int_{\Omega} v_{\varepsilon}^{\beta} \leq \int_{\Omega} v_{\varepsilon}(\cdot, t)+L_{g}|\Omega|
$$

hold for any $t>0$, which, in light of Lemma 3.2, proves (4.1). Going back to (4.3), we observe that integrating with respect to time immediately entails (4.2), concluding the proof.

In order to prepare for the core argument of our analysis in the next lemma we will require the following temporal cut-off functions. 


\section{Definition 4.2.}

Let $\xi_{0} \in C^{\infty}(\mathbb{R})$ be an arbitrary monotonically increasing function satisfying

$$
0 \leq \xi_{0} \leq 1 \text { on } \mathbb{R}, \quad \xi_{0} \equiv 0 \text { on }(-\infty, 0], \quad \text { and } \quad \xi_{0} \equiv 1 \text { on }(1, \infty) .
$$

For $T>0$ we then define

$$
\xi_{T}(t):=\xi_{0}(t-T), \quad t \in \mathbb{R} .
$$

The essential driving force of our analysis will be obtained by means of a well-known maximal Sobolev regularity estimate for the Neumann heat semigroup $([13,11])$ applied to the third equation of $(3.1)$. In our setting, relying on the result of Lemma 4.1 , we are hence able to obtain time-space bounds for the second derivatives of $w_{\varepsilon}$ in $L^{q}$ spaces with $1<q<\min \{\alpha, \beta\}$.

\section{Lemma 4.3.}

Let $\rho:=\min \{\alpha, \beta\}>1$. There exists $C>0$ such that for any $\varepsilon \in(0,1)$ and all $t \geq 0$ the solution $\left(u_{\varepsilon}, v_{\varepsilon}, w_{\varepsilon}\right)$ of (3.1) satisfies

$$
\int_{t}^{t+1}\left\|w_{\varepsilon}\right\|_{W^{2, \rho}(\Omega)}^{\rho} \leq C .
$$

Proof: For arbitrary fixed $\tau>0$ we let $\xi:=\xi_{\tau}$ denote the cut-off function introduced in Definition 4.2 and note that $\xi w_{\varepsilon}$ solves

$$
\begin{gathered}
\left(\xi w_{\varepsilon}\right)_{t}=\Delta\left(\xi w_{\varepsilon}\right)-\frac{\left(u_{\varepsilon}+v_{\varepsilon}\right)\left(\xi w_{\varepsilon}\right)}{1+\varepsilon\left(u_{\varepsilon}+v_{\varepsilon}\right) w_{\varepsilon}}-\mu\left(\xi w_{\varepsilon}\right)+\xi r(x, t)+\xi^{\prime} w_{\varepsilon} \quad \text { in } \Omega \times(\tau, \infty) \\
\text { with }\left(\xi w_{\varepsilon}\right)(\cdot, \tau)=0 \quad \text { in } \Omega \text { and } \nabla\left(\xi w_{\varepsilon}\right) \cdot \nu=0 \quad \text { on } \partial \Omega \times(\tau, \infty) .
\end{gathered}
$$

Hence, according to the maximal Sobolev regularity estimate for the Neumann heat semigroup $([13,11])$ for any $q>1$ there exists some $C_{1}>0$ such that for any $\varepsilon \in(0,1)$ the inequality

$$
\begin{aligned}
\int_{\tau}^{\tau+2}\left\|\left(\xi w_{\varepsilon}\right)_{t}\right\|_{L^{\rho}(\Omega)}^{\rho} & +\int_{\tau}^{\tau+2}\left\|\xi w_{\varepsilon}\right\|_{W^{2, \rho}(\Omega)}^{\rho} \\
& \leq C_{1}\left(0+\int_{\tau}^{\tau+2}\left\|-\frac{\left(u_{\varepsilon}+v_{\varepsilon}\right) \xi w_{\varepsilon}}{1+\varepsilon\left(u_{\varepsilon}+v_{\varepsilon}\right) w_{\varepsilon}}-\mu \xi w_{\varepsilon}+\xi r+\xi^{\prime} w_{\varepsilon}\right\|_{L^{\rho}(\Omega)}^{\rho}\right)
\end{aligned}
$$

holds. Since $\frac{1}{1+\varepsilon\left(u_{\varepsilon}+v_{\varepsilon}\right) w_{\varepsilon}} \leq 1$ on $\Omega \times(0, \infty)$, we can draw on the nonnegativity of $r, u_{\varepsilon}, v_{\varepsilon}$ and $w_{\varepsilon}$ on $\Omega \times(0, \infty)$, the fact that $\xi \leq 1$ on $\mathbb{R}$, Lemma 3.3 and (1.7) to obtain $C_{2}>0$ such that

$$
\begin{aligned}
\int_{\tau}^{\tau+2}\left\|\xi w_{\varepsilon}\right\|_{W^{2, \rho}(\Omega)}^{\rho} & \leq 2 C_{1}|\Omega|\left(\mu^{\rho} w_{*}^{\rho}+r_{*}^{\rho}+\left\|\xi^{\prime}\right\|_{L^{\infty}(\mathbb{R})}^{\rho} w_{*}^{\rho}\right)+C_{1} w_{*}^{q} \int_{\tau}^{\tau+2} \int_{\Omega}\left(u_{\varepsilon}+v_{\varepsilon}\right)^{\rho} \\
& \leq C_{2}+C_{2} \int_{\tau}^{\tau+2} \int_{\Omega} u_{\varepsilon}^{\rho}+C_{2} \int_{\tau}^{\tau+2} \int_{\Omega} v_{\varepsilon}^{\rho} \quad \text { for all } \varepsilon \in(0,1) .
\end{aligned}
$$

Here, we rely on (4.1) from Lemma 4.1 to find that

$$
\int_{\tau}^{\tau+2}\left\|\xi w_{\varepsilon}\right\|_{W^{2, \rho}(\Omega)}^{\rho} \leq C_{2}+2 C_{2}\left(\frac{L_{f}|\Omega|}{K_{f}}+\frac{u_{*}}{K_{f}}+\frac{L_{g}|\Omega|}{K_{g}}+\frac{v_{*}}{K_{g}}\right),
$$

which, due to the arbitrary choice of $\tau>0$ and the fact that $\xi \equiv 1$ on $(\tau+1, \tau+2)$, implies the existence of $C_{3}>0$ such that

$$
\int_{t}^{t+1}\left\|w_{\varepsilon}\right\|_{W^{2, \rho}(\Omega)}^{\rho} \leq C_{3}
$$

holds for each $\varepsilon \in(0,1)$ and all $t>1$. We are left with treating $t \in[0,1]$. Arguing along similar lines, this time investigating (3.1) directly without the cut-off and additionally relying on (1.8) to treat the first term, we find $C_{4}>0$ such that for any $\varepsilon \in(0,1)$

$$
\int_{0}^{2}\left\|w_{\varepsilon}\right\|_{W^{2, \rho}(\Omega)}^{\rho} \leq C_{1}\left\|w_{0}\right\|_{W^{2, \rho}(\Omega)}^{\rho}+2 C_{1}|\Omega|\left(\mu^{\rho} w_{*}^{\rho}+r_{*}^{\rho}\right)+C_{1} w_{*}^{\rho} \int_{0}^{2} \int_{\Omega}\left(u_{\varepsilon}+v_{\varepsilon}\right)^{\rho}
$$




$$
\leq C_{4}+C_{2} \int_{0}^{2} \int_{\Omega}\left(u_{\varepsilon}^{\rho}+v_{\varepsilon}^{\rho}\right) \leq C_{4}+2 C_{2}\left(\frac{L_{f}|\Omega|}{K_{f}}+\frac{u_{*}}{K_{f}}+\frac{L_{g}|\Omega|}{K_{g}}+\frac{v_{*}}{K_{g}}\right),
$$

completing the proof upon obvious choice for $C>0$.

In the next step we will see that the information on the Laplacian of $w_{\varepsilon}$, contained in Lemma 4.3 , is the key ingredient when making use of testing procedures to derive $L^{q}$ bounds for $u_{\varepsilon}$. The following lemma is also the crucial point where the conditions on $\alpha$ and $\min \{\alpha, \beta\}$ featured in Theorem 1.1 are first required.

Lemma 4.4.

Assume $\alpha>1+\sqrt{2}$ and $\beta>1$ and suppose that $\rho:=\min \{\alpha, \beta\}$ satisfies $\rho>\frac{\alpha+1}{\alpha-1}$. Then for any $q \in[2,(\alpha-1)(\rho-1))$ there exists $C>0$ such that for all $\varepsilon \in(0,1)$ the solution $\left(u_{\varepsilon}, v_{\varepsilon}, w_{\varepsilon}\right)$ of $(3.1)$ satisfies

$$
\frac{1}{q} \frac{\mathrm{d}}{\mathrm{d} t} \int_{\Omega} u_{\varepsilon}^{q}+(q-1) \int_{\Omega} u_{\varepsilon}^{q-2}\left|\nabla u_{\varepsilon}\right|^{2}+\frac{K_{f}}{2} \int_{\Omega} u_{\varepsilon}^{q+\alpha-1} \leq C \int_{\Omega}\left|\Delta w_{\varepsilon}\right|^{\rho}+C
$$

on $(0, \infty)$.

Proof: First note that, due to the assumption that $\rho>\frac{\alpha+1}{\alpha-1}$, the interval $I:=[2,(\alpha-1)(\rho-1))$ is not empty. Now, given any $q \in I$ we test the first equation of (3.1) against $u_{\varepsilon}^{q-1}$ and integrate by parts, which implies that

$$
\frac{1}{q} \frac{\mathrm{d}}{\mathrm{d} t} \int_{\Omega} u_{\varepsilon}^{q}=-(q-1) \int_{\Omega} u_{\varepsilon}^{q-2}\left|\nabla u_{\varepsilon}\right|^{2}+(q-1) \int_{\Omega} u_{\varepsilon}^{q-1}\left(\nabla u_{\varepsilon} \cdot \nabla w_{\varepsilon}\right)+\int_{\Omega} u_{\varepsilon}^{q-1} f\left(u_{\varepsilon}\right)
$$

holds on $(0, \infty)$, due to the prescribed boundary conditions. In light of the pointwise identity $u_{\varepsilon}^{q-1} \nabla u_{\varepsilon}=$ $\frac{1}{q} \nabla\left(u_{\varepsilon}^{q}\right)$ we may integrate by parts once more in the second term on the right to obtain

$$
\frac{1}{q} \frac{\mathrm{d}}{\mathrm{d} t} \int_{\Omega} u_{\varepsilon}^{q}=-(q-1) \int_{\Omega} u_{\varepsilon}^{q-2}\left|\nabla u_{\varepsilon}\right|^{2}-\frac{q-1}{q} \int_{\Omega} u_{\varepsilon}^{q} \Delta w_{\varepsilon}+\int_{\Omega} u_{\varepsilon}^{q-1} f\left(u_{\varepsilon}\right)
$$

is valid on $(0, \infty)$. Here, we make use of the nonnegativity of $u_{\varepsilon},(1.6)$ and Young's inequality to find $C_{1}>0$ such that for any $\varepsilon \in(0,1)$

$$
\frac{1}{q} \frac{\mathrm{d}}{\mathrm{d} t} \int_{\Omega} u_{\varepsilon}^{q} \leq-(q-1) \int_{\Omega} u_{\varepsilon}^{q-2}\left|\nabla u_{\varepsilon}\right|^{2}+C_{1} \int_{\Omega}\left|\Delta w_{\varepsilon}\right|^{\rho}+\int_{\Omega} u_{\varepsilon}^{\frac{q \rho}{\rho-1}}-K_{f} \int_{\Omega} u_{\varepsilon}^{q+\alpha-1}+L_{f} \int_{\Omega} u_{\varepsilon}^{q-1}
$$

on $(0, \infty)$. Since $\alpha$ is nonnegative and $q<(\alpha-1)(\rho-1)$ implies that also $\frac{q \rho}{\rho-1}<q-1+\alpha$ we may employ Young's inequality once again to the third and last terms on the right and conclude that there is some $C_{2}>0$ such that for any $\varepsilon \in(0,1)$ the inequality

$$
\frac{1}{q} \frac{\mathrm{d}}{\mathrm{d} t} \int_{\Omega} u_{\varepsilon}^{q}+(q-1) \int_{\Omega} u_{\varepsilon}^{q-2}\left|\nabla u_{\varepsilon}\right|^{2}+\frac{K_{f}}{2} \int_{\Omega} u_{\varepsilon}^{q+\alpha-1} \leq C_{1} \int_{\Omega}\left|\Delta w_{\varepsilon}\right|^{\rho}+C_{2}
$$

is satisfied on $(0, \infty)$.

In order to turn this differential inequality into some boundedness information we require the following supplementary lemma. This lemma is taken from [19, Lemma 3.4], where also additional details on the proof can be found.

\section{Lemma 4.5.}

For some $T \in(0, \infty]$ let $y \in C^{1}((0, T)) \cap C^{0}([0, T)), h \in C^{0}([0, T)), h \geq 0, C>0, a>0$ satisfy

$$
y^{\prime}(t)+a y(t) \leq h(t), \quad \int_{(t-1)_{+}}^{t} h(s) \mathrm{d} s \leq C
$$

for all $t \in(0, T)$. Then $y \leq y(0)+\frac{C}{1-e^{-a}}$ throughout $(0, T)$.

A combination of Lemmas 4.4 and 4.5 with the boundedness of $\int_{t}^{t+1} \int_{\Omega}\left|\Delta w_{\varepsilon}\right|^{\rho}$ provided by Lemma 4.3 now entails uniform (spatio-temporal) bounds for $u_{\varepsilon}$ in some $L^{q}$ spaces, where the admissible values for $q$ are already sufficiently large for our later purposes. 


\section{Lemma 4.6.}

Assume $\alpha>1+\sqrt{2}$ and $\beta>1$ and suppose that $\rho:=\min \{\alpha, \beta\}$ satisfies $\rho>\frac{\alpha+1}{\alpha-1}$. Then for any $q \in[2,(\alpha-1)(\rho-1))$ there exists $C>0$ such that for all $\varepsilon \in(0,1)$ the solution $\left(u_{\varepsilon}, v_{\varepsilon}, w_{\varepsilon}\right)$ of $(3.1)$ fulfills

$$
\int_{\Omega} u_{\varepsilon}^{q}(\cdot, t)+\int_{t}^{t+1} \int_{\Omega} u_{\varepsilon}^{q-2}\left|\nabla u_{\varepsilon}\right|^{2}+\int_{t}^{t+1} \int_{\Omega} u_{\varepsilon}^{q+\alpha-1} \leq C
$$

for all $t>0$.

Proof: From Lemma 4.4 we obtain $C_{1}>0$ such that

$$
\frac{1}{q} \frac{\mathrm{d}}{\mathrm{d} t} \int_{\Omega} u_{\varepsilon}^{q}+(q-1) \int_{\Omega} u_{\varepsilon}^{q-2}\left|\nabla u_{\varepsilon}\right|^{2}+\frac{K_{f}}{2} \int_{\Omega} u_{\varepsilon}^{q+\alpha-1} \leq C_{1} \int_{\Omega}\left|\Delta w_{\varepsilon}\right|^{\rho}+C_{1}
$$

is valid on $(0, \infty)$ for any $\varepsilon \in(0,1)$. To derive an inequality of the form featured in Lemma 4.5 from this, we note that by Lemma 4.3 there is $C_{2}>0$ satisfying

$$
\int_{t}^{t+1} \int_{\Omega}\left|\Delta w_{\varepsilon}\right|^{\rho} \leq C_{2} \quad \text { for all } \varepsilon \in(0,1) \text { and } t>0
$$

and that $\alpha>1+\sqrt{2}$ and Young's inequality entail the existence of $C_{3}=C_{3}\left(q, \alpha, K_{f}, \Omega\right)>0$ such that $\frac{1}{q} \int_{\Omega} u_{\varepsilon}^{q}(\cdot, t) \leq \frac{K_{f}}{2} \int_{\Omega} u_{\varepsilon}^{q+\alpha-1}(\cdot, t)+C_{3}$ for all $t>0$. Hence, setting $y_{\varepsilon}(t):=\frac{1}{q} \int_{\Omega} u_{\varepsilon}^{q}(\cdot, t), t>0$, we have

$$
y_{\varepsilon}^{\prime}(t)+y_{\varepsilon}(t) \leq C_{1} \int_{\Omega}\left|\Delta w_{\varepsilon}\right|^{\rho}+C_{1}+C_{3} \quad \text { for all } \varepsilon \in(0,1) \text { and } t>0,
$$

where we used the nonnegativity of $u_{\varepsilon}$ to drop the term containing the derivative. Now we can apply Lemma 4.5 in conjunction with (4.5) to obtain $C_{4}>0$ such that

$$
\int_{\Omega} u_{\varepsilon}^{q}(\cdot, t) \leq C_{4} \quad \text { for all } \varepsilon \in(0,1) \text { and } t>0
$$

Finally, we see from (4.4) upon integration over $(t, t+1)$ that

$$
\frac{1}{q} \int_{\Omega} u_{\varepsilon}^{q}(\cdot, t+1)+(q-1) \int_{t}^{t+1} \int_{\Omega} u_{\varepsilon}^{q-2}\left|\nabla u_{\varepsilon}\right|^{2}+\frac{K_{f}}{2} \int_{t}^{t+1} \int_{\Omega} u_{\varepsilon}^{q+\alpha-1} \leq C_{4}+C_{1} C_{2}+C_{1} \quad \text { for all } t>0,
$$

concluding the proof.

An evident consequence of the lemma above are the following bounds.

\section{Corollary 4.7.}

Assume $\alpha>1+\sqrt{2}$ and $\beta>1$ and suppose that $\rho:=\min \{\alpha, \beta\}$ satisfies $\rho>\frac{\alpha+1}{\alpha-1}$. Then there exists $C>0$ such that for all $\varepsilon \in(0,1)$ the solution $\left(u_{\varepsilon}, v_{\varepsilon}, w_{\varepsilon}\right)$ of $(3.1)$ fulfills

$$
\int_{\Omega} u_{\varepsilon}^{2}(\cdot, t)+\int_{t}^{t+1} \int_{\Omega}\left|\nabla u_{\varepsilon}\right|^{2}+\int_{t}^{t+1} \int_{\Omega} u_{\varepsilon}^{\alpha+1} \leq C
$$

for all $t>0$.

Proof: This follows immediately from Lemma 4.6 when applied for $q=2$.

To establish the uniform bound for $u_{\varepsilon}$ in $L^{2}(\Omega)$ we made essential use of the knowledge that $\Delta w_{\varepsilon}$ is uniformly bounded in $L^{\rho}(\Omega \times(t, t+1))$. Since up to this point the only regularity information on any derivative of $u_{\varepsilon}$ is a mere bound on $\nabla u_{\varepsilon}$ in $L^{2}(\Omega \times(t, t+1))$, it is therefore not surprising that the treatment of $v_{\varepsilon}$ is exceedingly difficult and we cannot expect standard testing measures to improve our knowledge on $v_{\varepsilon}$ beyond the result of Lemma 4.1. Hence, we turn to the investigation of the rather mild expression $\ln \left(v_{\varepsilon}+1\right)$, which will at least provide minimal information on the spatial derivative of $v_{\varepsilon}$ in the sense of the following lemma. 


\section{Lemma 4.8 .}

Assume $\alpha>1+\sqrt{2}$ and $\beta>1$ and suppose that $\rho:=\min \{\alpha, \beta\}$ satisfies $\rho>\frac{\alpha+1}{\alpha-1}$. Then for any $T>0$ there exists $C(T)>0$ such that for all $\varepsilon \in(0,1)$ the solution $\left(u_{\varepsilon}, v_{\varepsilon}, w_{\varepsilon}\right)$ of $(3.1)$ satisfies

$$
\int_{0}^{T} \int_{\Omega} \frac{\left|\nabla v_{\varepsilon}\right|^{2}}{\left(v_{\varepsilon}+1\right)^{2}} \leq C(T) \text {. }
$$

Proof: Straightforward calculations using the second equation of (3.1) and integration by parts show that

$$
\begin{aligned}
-\frac{\mathrm{d}}{\mathrm{d} t} \int_{\Omega} \ln \left(v_{\varepsilon}+1\right) & =-\int_{\Omega} \frac{\Delta v_{\varepsilon}-\nabla \cdot\left(v_{\varepsilon} \nabla u_{\varepsilon}\right)+g\left(v_{\varepsilon}\right)}{v_{\varepsilon}+1} \\
& =-\int_{\Omega} \frac{\left|\nabla v_{\varepsilon}\right|^{2}}{\left(v_{\varepsilon}+1\right)^{2}}+\int_{\Omega} \frac{v_{\varepsilon}}{\left(v_{\varepsilon}+1\right)^{2}}\left(\nabla v_{\varepsilon} \cdot \nabla u_{\varepsilon}\right)-\int_{\Omega} \frac{g\left(v_{\varepsilon}\right)}{v_{\varepsilon}+1}
\end{aligned}
$$

is valid for all $t>0$. Since $v_{\varepsilon}$ is nonnegative, an application of Young's inequality and (1.6) thereby entails that

$$
\begin{aligned}
-\frac{\mathrm{d}}{\mathrm{d} t} \int_{\Omega} \ln \left(v_{\varepsilon}+1\right)+\frac{1}{2} \int_{\Omega} \frac{\left|\nabla v_{\varepsilon}\right|^{2}}{\left(1+v_{\varepsilon}\right)^{2}} & \leq-\int_{\Omega} g\left(v_{\varepsilon}\right)+\frac{1}{2} \int_{\Omega} \frac{v_{\varepsilon}^{2}}{\left(1+v_{\varepsilon}\right)^{2}}\left|\nabla u_{\varepsilon}\right|^{2} \\
& \leq k_{g} \int_{\Omega} v_{\varepsilon}^{\beta}+l_{g}|\Omega|+\frac{1}{2} \int_{\Omega}\left|\nabla u_{\varepsilon}\right|^{2} \text { for all } t>0 .
\end{aligned}
$$

Given any $T>0$, we can now integrate this inequality with respect to time and immediately obtain that

$$
\frac{1}{2} \int_{0}^{T} \int_{\Omega} \frac{\left|\nabla v_{\varepsilon}\right|^{2}}{\left(v_{\varepsilon}+1\right)^{2}} \leq \int_{\Omega} \ln \left(v_{\varepsilon}(\cdot, T)+1\right)+l_{g}|\Omega| T+k_{g} \int_{0}^{T} \int_{\Omega} v_{\varepsilon}^{\beta}+\int_{0}^{T} \int_{\Omega}\left|\nabla u_{\varepsilon}\right|^{2} .
$$

To further estimate the right hand side, we recall that Lemma 4.1 and Corollary 4.7 provide $C_{1}(T)>0$ such that $k_{g} \int_{0}^{T} \int_{\Omega} v_{\varepsilon}^{\beta}+\int_{0}^{T} \int_{\Omega}\left|\nabla u_{\varepsilon}\right|^{2} \leq C_{1}(T)$ and that Lemma 3.2, when combined with the the evident estimate $0 \leq \ln (\xi+1) \leq \xi$ for all $\xi \geq 0$, entails $\int_{\Omega} \ln \left(v_{\varepsilon}(\cdot, T)+1\right) \leq \int_{\Omega} v_{\varepsilon}(\cdot, T) \leq v_{*}$. Plugging these bounds into (4.6) finally implies

$$
\frac{1}{2} \int_{0}^{T} \frac{\left|\nabla v_{\varepsilon}\right|^{2}}{\left(v_{\varepsilon}+1\right)^{2}} \leq v_{*}+l_{g}|\Omega| T+C_{1}(T),
$$

completing the proof.

\section{Refined spatio-temporal estimates and the regularity of time derivatives - Preparing convergence in suitable $L^{p}$ spaces}

The goal of this section is the preparation of uniform bounds in $L^{p}$ spaces for suitably chosen $p>1$. In the succeding sections we will combine these bounds with Vitali's theorem to attain the strong convergence properties necessary for passing to limit in the integrals appearing in integral identities corresponding to (2.1), (2.2) and (2.3) of the solution concept (see Section 7). Let us begin with a straightforward implication of Lemma 4.3.

Lemma 5.1.

Let $\rho:=\min \{\alpha, \beta\}>1$. There is $C>0$ such that for all $t>0$ and all $\varepsilon \in(0,1)$ the solution $\left(u_{\varepsilon}, v_{\varepsilon}, w_{\varepsilon}\right)$ of (3.1) satisfies

$$
\int_{t}^{t+1} \int_{\Omega}\left|\nabla w_{\varepsilon}\right|^{2 \rho} \leq C
$$

Proof: The Gagliardo-Nirenberg inequality asserts the existence of $C_{1}>0$ such that

$$
\|\phi\|_{W^{1,2 \rho}(\Omega)} \leq C_{1}\|\phi\|_{W^{2, \rho}(\Omega)}^{a}\|\phi\|_{L^{\infty}(\Omega)}^{1-a} \quad \text { for all } \phi \in W^{2, \rho}(\Omega),
$$


where $a=\frac{\frac{1}{2}-\frac{1}{2 \rho}}{1-\frac{1}{\rho}}=\frac{1}{2}$. Making use of Lemma 3.3 we thus obtain that $w_{\varepsilon}$ satisfies

$$
\int_{t}^{t+1}\left\|w_{\varepsilon}\right\|_{W^{1,2 \rho}(\Omega)}^{2 \rho} \leq C_{1} \int_{t}^{t+1}\left\|w_{\varepsilon}\right\|_{W^{2, \rho}(\Omega)}^{\rho}\left\|w_{\varepsilon}\right\|_{L^{\infty}(\Omega)}^{\rho} \leq C_{1} w_{*}^{\rho} \int_{t}^{t+1}\left\|w_{\varepsilon}\right\|_{W^{2, \rho}(\Omega)}^{\rho}
$$

for all $t>0$ and all $\varepsilon \in(0,1)$. In light of Lemma 4.3 this entails the existence of $C_{2}>0$ such that

$$
\int_{t}^{t+1}\left\|w_{\varepsilon}\right\|_{W^{1,2 \rho}(\Omega)}^{2 \rho} \leq C_{2} \quad \text { for all } t>0 \text { and } \varepsilon \in(0,1)
$$

from which we immediately conclude the asserted bound.

Another direct implication of the bounds prepared at the beginning of Section 4 and the $L^{\infty}$ estimate for $w_{\varepsilon}$ is the following lemma.

Lemma 5.2.

Let $\rho:=\min \{\alpha, \beta\}>1$. For all $s \in(1, \rho)$ and $T>0$ there is $C(T)>0$ such that for all $\varepsilon \in(0,1)$ the solution $\left(u_{\varepsilon}, v_{\varepsilon}, w_{\varepsilon}\right)$ of (3.1) fulfills

$$
\int_{0}^{T} \int_{\Omega}\left(\frac{\left(u_{\varepsilon}+v_{\varepsilon}\right) w_{\varepsilon}}{1+\varepsilon\left(u_{\varepsilon}+v_{\varepsilon}\right) w_{\varepsilon}}\right)^{s} \leq C(T) .
$$

Proof: We fix any $s \in(1, \rho)$. Since $\frac{1}{\left(1+\varepsilon\left(u_{\varepsilon}+v_{\varepsilon}\right) w_{\varepsilon}\right)^{s}} \leq 1$ on $\Omega \times(0, \infty)$, we can make use of Young's inequality and Lemma 3.3 to estimate

$$
\int_{0}^{T} \int_{\Omega}\left(\frac{\left(u_{\varepsilon}+v_{\varepsilon}\right) w_{\varepsilon}}{1+\varepsilon\left(u_{\varepsilon}+v_{\varepsilon}\right) w_{\varepsilon}}\right)^{s} \leq \int_{0}^{T} \int_{\Omega}\left(u_{\varepsilon}+v_{\varepsilon}\right)^{\rho}+\int_{0}^{T} \int_{\Omega} w_{\varepsilon}^{\frac{s \rho}{\rho-s}} \leq 2^{\rho} \int_{0}^{T} \int_{\Omega} u_{\varepsilon}^{\rho}+2^{\rho} \int_{0}^{T} \int_{\Omega} v_{\varepsilon}^{\rho}+w_{*}^{\frac{s \rho}{\rho-s}}|\Omega| T .
$$

The boundedness of the remaining terms is a direct consequence of Lemma 4.1 due to $\rho=\min \{\alpha, \beta\}$.

In the next lemma we will take a look at bounds targeting $f\left(u_{\varepsilon}\right)$ and $g\left(v_{\varepsilon}\right)$. Here we note that, in particular, the uniform bound for $f\left(u_{\varepsilon}\right)$ in $L^{\frac{(\alpha+1) s}{\alpha}}(\Omega \times(0, T))$ for some $s>1$ is one essential part in order to later refine the convergence result for $\nabla u_{\varepsilon}$ to strong convergence in $L^{2}(\Omega \times(0, T))$ (cf. Section 7 ), which is also the main reason we had to treat the permissible exponents in Lemma 4.4 so carefully.

\section{Lemma 5.3.}

Assume $\alpha>1+\sqrt{2}$ and $\beta>1$ and suppose that $\rho:=\min \{\alpha, \beta\}$ satisfies $\rho>\frac{\alpha+1}{\alpha-1}$. Then there exists $s>1$ such that for any $T>0$ there is $C(T)>0$ such that for all $\varepsilon \in(0,1)$ the solution $\left(u_{\varepsilon}, v_{\varepsilon}, w_{\varepsilon}\right)$ of (3.1) fulfills

$$
\int_{0}^{T} \int_{\Omega}\left|f\left(u_{\varepsilon}\right)\right|^{\frac{\alpha+1}{\alpha} s}+\int_{0}^{T} \int_{\Omega}\left|\frac{g\left(v_{\varepsilon}\right)}{v_{\varepsilon}+1}\right|^{s} \leq C(T) .
$$

Proof: We set $s_{0}:=\min \left\{\frac{\alpha-1}{\alpha+1} \rho, \frac{\beta}{\beta-1}\right\}$ and note that the conditions $\rho>\frac{\alpha+1}{\alpha-1}$ and $\beta>1$ clearly imply that $s_{0}>1$. Hence, we can pick some $s \in\left(1, s_{0}\right)$ and note that since $(\alpha+1) s<(\alpha-1) \rho$ and $(\beta-1) s<\beta$ we can rely on Lemma 4.6, employed for $q=(\alpha+1) s-(\alpha-1)<(\alpha-1)(\rho-1)$, and Lemma 4.1 to obtain $C_{1}(T)>0$ such that

$$
\int_{0}^{T} \int_{\Omega} u_{\varepsilon}^{(\alpha+1) s}+\int_{0}^{T} \int_{\Omega} v_{\varepsilon}^{(\beta-1) s} \leq C_{1}(T) \text { for all } \varepsilon \in(0,1) .
$$

Now, making use of (1.6) we find $C_{2}>0$ such that

$$
\int_{0}^{T} \int_{\Omega}\left|f\left(u_{\varepsilon}\right)\right|^{\frac{\alpha+1}{\alpha} s}+\int_{0}^{T} \int_{\Omega}\left|\frac{g\left(v_{\varepsilon}\right)}{v_{\varepsilon}+1}\right|^{s} \leq C_{2} \int_{0}^{T} \int_{\Omega} u_{\varepsilon}^{(\alpha+1) s}+C_{2} \int_{0}^{T} \int_{\Omega} \frac{v_{\varepsilon}^{\beta s}}{\left(v_{\varepsilon}+1\right)^{s}}+C_{2} T
$$

holds for any $T>0$ and all $\varepsilon \in(0,1)$. Relying on (5.1) and the fact that $\xi^{\beta s}(\xi+1)^{-s} \leq \xi^{(\beta-1) s}$ for all $\xi \geq 0$ we find that

$$
\int_{0}^{T} \int_{\Omega}\left|f\left(u_{\varepsilon}\right)\right|^{\frac{\alpha+1}{\alpha} s}+\int_{0}^{T} \int_{\Omega}\left|\frac{g\left(v_{\varepsilon}\right)}{v_{\varepsilon}+1}\right|^{s} \leq C_{2} C_{1}(T)+C_{2} C_{1}(T)+C_{2} T
$$

is valid for any $T>0$ and all $\varepsilon \in(0,1)$. 
Making use of Corollary 4.7 and Lemma 5.1 we can also readily attain a $\varepsilon$-uniform bound on $u_{\varepsilon} \nabla w_{\varepsilon}$ in $L^{s}(\Omega \times(0, T))$ for some $s>2$. This result will also be the second essential part for establishing the strong convergence of $\nabla u_{\varepsilon}$ in Section 7 .

\section{Lemma 5.4.}

Assume $\alpha>1+\sqrt{2}$ and $\beta>1$ and suppose that $\rho:=\min \{\alpha, \beta\}$ satisfies $\rho>\frac{\alpha+1}{\alpha-1}$. Then there exists some $s>2$ such that for any $T>0$ there is $C(T)>0$ such that for all $\varepsilon \in(0,1)$ the solutions $\left(u_{\varepsilon}, v_{\varepsilon}, w_{\varepsilon}\right)$ of (3.1) satisfies

$$
\int_{0}^{T} \int_{\Omega}\left|u_{\varepsilon} \nabla w_{\varepsilon}\right|^{s} \leq C(T)
$$

Proof: We note that due to $\rho>\frac{\alpha+1}{\alpha-1}$ the interval $I:=\left(2, \frac{2 \rho(\alpha+1)}{\alpha+1+2 \rho}\right)$ is not empty and that for $s \in I$ we have $s<\alpha+1$ and $\frac{(\alpha+1) s}{\alpha+1-s}<2 \rho$. Accordingly, for any fixed $s \in I$ straightforward applications of Young's inequality entail the existence of $C_{1}>0$ such that

$$
\int_{0}^{T} \int_{\Omega} u_{\varepsilon}^{s}\left|\nabla w_{\varepsilon}\right|^{s} \leq \int_{0}^{T} \int_{\Omega} u_{\varepsilon}^{\alpha+1}+\int_{0}^{T} \int_{\Omega}\left|\nabla w_{\varepsilon}\right|^{\frac{(\alpha+1) s}{\alpha+1-s}} \leq \int_{0}^{T} \int_{\Omega} u_{\varepsilon}^{\alpha+1}+\int_{0}^{T} \int_{\Omega}\left|\nabla w_{\varepsilon}\right|^{2 \rho}+C_{1} T
$$

holds for any $T>0$ and all $\varepsilon \in(0,1)$. In light of the previously prepared bounds of Corollary 4.7 and Lemma 5.1 we can immediately conclude the assertion.

Before we can employ an Aubin-Lions type argument to construct limit objects, which will build the basis for our convergence results, we still require some information on the regularity of the time derivatives of the approximate solutions.

\section{Lemma 5.5.}

Assume $\alpha>1+\sqrt{2}$ and $\beta>1$ and suppose that $\rho:=\min \{\alpha, \beta\}$ satisfies $\rho>\frac{\alpha+1}{\alpha-1}$. For every $T>0$ exists $C(T)>0$ such that for any $\varepsilon \in(0,1)$ the solution $\left(u_{\varepsilon}, v_{\varepsilon}, w_{\varepsilon}\right)$ of $(3.1)$ fulfills

$$
\int_{0}^{T}\left\|u_{\varepsilon t}\right\|_{\left(W^{2,2}(\Omega)\right)^{*}}+\int_{0}^{T}\left\|\partial_{t} \ln \left(v_{\varepsilon}+1\right)\right\|_{\left(W^{2,2}(\Omega)\right)^{*}}+\int_{0}^{T}\left\|w_{\varepsilon t}\right\|_{\left(W^{2,2}(\Omega)\right)^{*}} \leq C(T) .
$$

Proof: For fixed $\psi \in C^{\infty}(\bar{\Omega})$ we test the first equation of (3.1) against $\psi$ and estimate by means of the Hölder and Young inequalities that for all $\varepsilon \in(0,1)$

$$
\begin{aligned}
\left|\int_{\Omega} u_{\varepsilon t} \cdot \psi\right| & =\left|-\int_{\Omega} \nabla u_{\varepsilon} \cdot \nabla \psi+\int_{\Omega} u_{\varepsilon} \nabla w_{\varepsilon} \cdot \nabla \psi+\int_{\Omega} f\left(u_{\varepsilon}\right) \psi\right| \\
& \leq\left(\left\|\nabla u_{\varepsilon}\right\|_{L^{2}(\Omega)}^{2}+\left\|u_{\varepsilon} \nabla w_{\varepsilon}\right\|_{L^{2}(\Omega)}^{2}+\frac{1}{2}\right)\|\nabla \psi\|_{L^{2}(\Omega)}+\left\|f\left(u_{\varepsilon}\right)\right\|_{L^{1}(\Omega)}\|\psi\|_{L^{\infty}(\Omega)}
\end{aligned}
$$

is valid on $(0, \infty)$. Making use of the embedding $W^{2,2}(\Omega) \hookrightarrow L^{\infty}(\Omega)$ we can hence find $C_{1}>0$ such that for all $\varepsilon \in(0,1)$

$$
\int_{0}^{T}\left\|u_{\varepsilon t}\right\|_{\left(W^{2,2}(\Omega)\right)^{*}} \leq C_{1} \int_{0}^{T}\left(\left\|\nabla u_{\varepsilon}\right\|_{L^{2}(\Omega)}^{2}+\left\|u_{\varepsilon} \nabla w_{\varepsilon}\right\|_{L^{2}(\Omega)}^{2}+\left\|f\left(u_{\varepsilon}\right)\right\|_{L^{1}(\Omega)}+\frac{1}{2}\right),
$$

which entails the boundedness of the first quantity appearing in (5.2) in light of Corollary 4.7 and Lemmas 5.4 and 5.3. Arguing similarly for the second component of (3.1) we again fix $\psi \in C^{\infty}(\bar{\Omega})$ and find that for all $\varepsilon \in(0,1)$

$$
\begin{aligned}
& \left|\int_{\Omega} \psi \partial_{t} \ln \left(v_{\varepsilon}+1\right)\right| \\
= & \left|\int_{\Omega} \frac{\left|\nabla v_{\varepsilon}\right|^{2}}{\left(v_{\varepsilon}+1\right)^{2}} \psi-\int_{\Omega} \frac{\nabla v_{\varepsilon} \cdot \nabla \psi}{v_{\varepsilon}+1}-\int_{\Omega} \frac{v_{\varepsilon}\left(\nabla u_{\varepsilon} \cdot \nabla v_{\varepsilon}\right)}{\left(v_{\varepsilon}+1\right)^{2}} \psi+\int_{\Omega} \frac{v_{\varepsilon}\left(\nabla u_{\varepsilon} \cdot \nabla \psi\right)}{v_{\varepsilon}+1}+\int_{\Omega} \frac{g\left(v_{\varepsilon}\right)}{v_{\varepsilon}+1} \psi\right| \\
\leq & \left(\frac{5}{4} \int_{\Omega} \frac{\left|\nabla v_{\varepsilon}\right|^{2}}{\left(v_{\varepsilon}+1\right)^{2}}+\int_{\Omega}\left|\nabla u_{\varepsilon}\right|^{2}+\int_{\Omega}\left|\frac{g\left(v_{\varepsilon}\right)}{v_{\varepsilon}+1}\right|\right)\|\psi\|_{L^{\infty}(\Omega)}+\left(\int_{\Omega} \frac{\left|\nabla v_{\varepsilon}\right|^{2}}{\left(v_{\varepsilon}+1\right)^{2}}+\int_{\Omega}\left|\nabla u_{\varepsilon}\right|^{2}+\frac{1}{2}\right)\|\nabla \psi\|_{L^{2}(\Omega)}
\end{aligned}
$$


holds on $(0, \infty)$. And that hence, there is $C_{2}>0$ such that for any $T>0$ and all $\varepsilon \in(0,1)$

$$
\int_{0}^{T}\left\|\partial_{t} \ln \left(v_{\varepsilon}+1\right)\right\|_{\left(W^{2,2}(\Omega)\right)^{*}} \leq C_{2} \int_{0}^{T}\left(\int_{\Omega} \frac{\left|\nabla v_{\varepsilon}\right|^{2}}{\left(v_{\varepsilon}+1\right)^{2}}+\int_{\Omega}\left|\nabla u_{\varepsilon}\right|^{2}+\int_{\Omega}\left|\frac{g\left(v_{\varepsilon}\right)}{v_{\varepsilon}+1}\right|+1\right),
$$

from which the boundedness of the second part of (5.2) follows as a consequence of Lemma 4.8, Corollary 4.7 and Lemma 5.3. Finally, we reiterate the arguments once more for the last equations of (3.1), where for fixed $\psi \in C^{\infty}(\bar{\Omega})$ and all $\varepsilon \in(0,1)$, by Hölder's and Young's inequalities and Lemma 3.3,

$$
\begin{aligned}
\left|\int_{\Omega} w_{\varepsilon t} \psi\right| & =\left|-\int_{\Omega} \nabla w_{\varepsilon} \cdot \nabla \psi-\int_{\Omega} \frac{\left(u_{\varepsilon}+v_{\varepsilon}\right) w_{\varepsilon} \psi}{1+\varepsilon\left(u_{\varepsilon}+v_{\varepsilon}\right) w_{\varepsilon}}-\mu \int_{\Omega} w_{\varepsilon} \psi+\int_{\Omega} r \psi\right| \\
& \leq\left(\int_{\Omega}\left|\nabla w_{\varepsilon}\right|^{2}+\frac{1}{4}\right)\|\nabla \psi\|_{L^{2}(\Omega)}+\left(w_{*} \int_{\Omega} u_{\varepsilon}+w_{*} \int_{\Omega} v_{\varepsilon}+\mu w_{*}|\Omega|+r_{*}|\Omega|\right)\|\psi\|_{L^{\infty}(\Omega)}
\end{aligned}
$$

is valid on $(0, \infty)$. Hence, the embedding $W^{2,2}(\Omega) \hookrightarrow L^{\infty}(\Omega)$ readily entails the existence of $C_{3}>0$ such that for any $T>0$ and all $\varepsilon \in(0,1)$ we have

$$
\int_{0}^{T}\left\|w_{\varepsilon t}\right\|_{\left(W^{2,2}(\Omega)\right)^{*}} \leq C_{3} \int_{0}^{T}\left(\int_{\Omega}\left|\nabla w_{\varepsilon}\right|^{2}+\int_{\Omega} u_{\varepsilon}+\int_{\Omega} v_{\varepsilon}+1\right),
$$

finalizing the proof in light of Lemmas 5.1 and 3.2 .

\section{Construction of limit functions}

With the $\varepsilon$-uniform bounds prepared in the previous sections we can now derive the existence of limit functions $u, v, w$ satisfying the regularity conditions appearing in Definition 2.1. These uniform bounds, moreover, provide various precompactness properties from which we obtain a list of convergences, which will be of importance when proceeding to prove the inequality and equalities prescribed by $(2.1),(2.2)$ and (2.3).

\section{Proposition 6.1.}

Assume $\alpha>1+\sqrt{2}$ and $\beta>1$ and suppose that $\rho:=\min \{\alpha, \beta\}$ satisfies $\rho>\frac{\alpha+1}{\alpha-1}$. Then there exist a sequence $\left(\varepsilon_{j}\right)_{j \in N} \subset(0,1)$ with $\varepsilon_{j} \searrow 0$ as $j \rightarrow \infty$ and functions

$$
\begin{aligned}
& u \in L^{\infty}\left((0, \infty) ; L^{1}(\Omega)\right) \cap L_{l o c}^{\alpha+1}(\bar{\Omega} \times[0, \infty)) \quad \text { with } \quad \nabla u \in L_{l o c}^{2}\left(\bar{\Omega} \times[0, \infty) ; \mathbb{R}^{2}\right), \\
& v \in L^{\infty}\left((0, \infty) ; L^{1}(\Omega)\right) \cap L_{l o c}^{\beta}(\bar{\Omega} \times[0, \infty)) \quad \text { with } \quad \nabla \ln (v+1) \in L_{l o c}^{2}\left(\bar{\Omega} \times[0, \infty) ; \mathbb{R}^{2}\right), \\
& w \in L^{\infty}(\Omega \times(0, \infty)) \cap L_{l o c}^{\rho}\left([0, \infty) ; W^{2, \rho}(\Omega)\right) \quad \text { with } \quad \nabla w \in L_{l o c}^{2}\left(\bar{\Omega} \times[0, \infty) ; \mathbb{R}^{2}\right),
\end{aligned}
$$

such that

$$
f(u) \in L_{l o c}^{\frac{\alpha+1}{\alpha}}(\bar{\Omega} \times[0, \infty)) \quad \text { and } \quad \frac{g(v)}{v+1} \in L_{l o c}^{\frac{\beta}{\beta-1}}(\bar{\Omega} \times[0, \infty))
$$

and such that the solutions $\left(u_{\varepsilon}, v_{\varepsilon}, w_{\varepsilon}\right)$ of (3.1) fulfill

$$
\begin{aligned}
u_{\varepsilon} & \rightarrow u & & \text { in } L_{l o c}^{p}(\bar{\Omega} \times[0, \infty)) \text { for any } p \in[1, \alpha+1) \text { and a.e. in } \Omega \times(0, \infty), \\
u_{\varepsilon}(\cdot, t) & \rightarrow u(\cdot, t) & & \text { in } L^{2}(\Omega) \text { for a.e. } t>0, \\
\nabla u_{\varepsilon} & \rightarrow \nabla u & & \text { in } L_{l o c}^{2}\left(\bar{\Omega} \times[0, \infty) ; \mathbb{R}^{2}\right), \\
u_{\varepsilon} & \rightarrow u & & \text { in } L_{l o c}^{\alpha+1}(\bar{\Omega} \times[0, \infty)), \\
f\left(u_{\varepsilon}\right) & \rightarrow f(u) & & \text { in } L_{l o c}^{\frac{\alpha+1}{\alpha}}(\bar{\Omega} \times[0, \infty)), \\
v_{\varepsilon} & \rightarrow v & & \text { in } L_{l o c}^{p}(\bar{\Omega} \times[0, \infty)) \text { for any } p \in[1, \beta) \text { and a.e. in } \Omega \times(0, \infty), \\
\ln \left(v_{\varepsilon}+1\right) & \rightarrow \ln (v+1) & & \text { in } L_{l o c}^{2}\left([0, \infty) ; W^{1,2}(\Omega)\right), \\
v_{\varepsilon} & \rightarrow v & & \text { in } L_{l o c}^{\beta}(\bar{\Omega} \times[0, \infty)), \\
\frac{g\left(v_{\varepsilon}\right)}{v_{\varepsilon}+1} & \rightarrow \frac{g(v)}{v+1} & & \text { in } L_{l o c}^{1}(\bar{\Omega} \times[0, \infty))
\end{aligned}
$$




$$
\begin{aligned}
w_{\varepsilon} \rightarrow w & \text { in } L_{l o c}^{2}(\bar{\Omega} \times[0, \infty)) \text { and a.e. in } \Omega \times(0, \infty), \\
w_{\varepsilon} \stackrel{\star}{\rightarrow} w & \text { in } L^{\infty}(\Omega \times(0, \infty)), \\
w_{\varepsilon} \rightarrow w & \text { in } L_{l o c}^{\rho}\left([0, \infty) ; W^{2, \rho}(\Omega)\right), \\
\nabla w_{\varepsilon} \rightarrow \nabla w & \text { in } L_{l o c}^{2}\left(\bar{\Omega} \times[0, \infty) ; \mathbb{R}^{2}\right), \\
\frac{\left(u_{\varepsilon}+v_{\varepsilon}\right) w_{\varepsilon}}{1+\varepsilon\left(u_{\varepsilon}+v_{\varepsilon}\right) w_{\varepsilon}} \rightarrow(u+v) w & \text { in } L_{l o c}^{1}(\bar{\Omega} \times[0, \infty)), \\
u_{\varepsilon} \nabla w_{\varepsilon} \rightarrow u \nabla w & \text { in } L_{l o c}^{2}\left(\bar{\Omega} \times[0, \infty) ; \mathbb{R}^{2}\right),
\end{aligned}
$$

as $\varepsilon=\varepsilon_{j} \searrow 0$, and such that $u \geq 0, v \geq 0$ and $w \geq 0$ a.e. in $\Omega \times(0, \infty)$. Moreover, $u$ and $v$ satisfy

$$
\int_{\Omega} u(\cdot, t) \leq u_{*} \quad \text { and } \quad \int_{\Omega} v(\cdot, t) \leq v_{*} \quad \text { for a.e. } t>0
$$

as well as

$$
\int_{\Omega} v(\cdot, t) \leq \int_{\Omega} v_{0}+\int_{0}^{t} \int_{\Omega} g(v) \text { for a.e. } t>0 .
$$

Proof: As a consequence of Corollary 4.7 and Lemma $5.5\left\{u_{\varepsilon}\right\}_{\varepsilon \in(0,1)}$ is bounded in $L^{2}\left((0, T) ; W^{1,2}(\Omega)\right)$ with $\left\{u_{\varepsilon t}\right\}_{\varepsilon \in(0,1)}$ being bounded in $L^{1}\left((0, T) ;\left(W^{2,2}(\Omega)\right)^{*}\right)$ for any $T>0$. Thus, we may employ an Aubin-Lions type lemma (e.g. [29, Corollary 8.4]) and obtain that $\left\{u_{\varepsilon}\right\}_{\varepsilon \in(0,1)}$ is relatively precompact in $L^{2}\left((0, T) ; W^{1,2}(\Omega)\right)$ for any $T>0$. This ensures the existence of $u \in L_{l o c}^{2}\left([0, \infty) ; W^{1,2}(\Omega)\right)$ and a subsequence $\left(\varepsilon_{j}\right)_{j \in \mathbb{N}} \subset(0,1)$ satisfying $u_{\varepsilon} \rightarrow u$ a.e. in $\Omega \times(0, \infty)$ and in $L_{l o c}^{2}(\bar{\Omega} \times[0, \infty))$, which also implies (6.2). The weak convergences (if necessary along another non-relabeled subsequence) featured in (6.3) and (6.4) are then immediate consequences of the spatio-temporal bounds established in Corollary 4.7. The strong convergences appearing in (6.1) and (6.5) are then implied by Vitali's theorem, where for the equiintegrability of $\left\{\left|f\left(u_{\varepsilon_{j}}\right)\right|^{\frac{\alpha+1}{\alpha}}\right\}_{j \in \mathbb{N}}$ we additionally rely on Lemma 5.3. Similarly, a combination of Lemmas 4.8 and 5.5 with a Poincaré-type inequality, the basic estimate $0 \leq \ln (\xi+1) \leq \xi$ on $[0, \infty)$ and Lemma 3.2 entails the boundedness of $\left\{\ln \left(v_{\varepsilon}+1\right)\right\}_{\varepsilon \in(0,1)}$ in $L^{2}\left((0, T) ; W^{1,2}(\Omega)\right)$ and $\left\{v_{\varepsilon t}\right\}_{\varepsilon \in(0,1)}$ in $L^{1}\left((0, T) ;\left(W^{2,2}(\Omega)\right)^{*}\right)$ for any $T>0$, so that another application of the Aubin-Lions lemma provides a further subsequence along which $v_{\varepsilon} \rightarrow v$ a.e. in $\Omega \times(0, \infty)$, from which, again due to the boundedness of $\left\{\ln \left(v_{\varepsilon}+1\right)\right\}_{\varepsilon \in(0,1)}$ in $L^{2}\left((0, T) ; W^{1,2}(\Omega)\right)$, we can also conclude that $(6.7)$ holds. The precompactness properties contained in the the bounds provided by Lemmas 4.1 and 5.3 , the fact that $\frac{\beta}{\beta-1}>1$ and Vitali's theorem then entail the properties listed in (6.6), (6.8) and (6.9). Likewise we note that Lemmas 3.3, 5.1 and 5.5 imply that $\left\{w_{\varepsilon}\right\}_{\varepsilon \in(0,1)}$ is bounded in $L^{2}\left((0, T) ; W^{1,2}(\Omega)\right)$ with $\left\{w_{\varepsilon t}\right\}_{\varepsilon \in(0,1)}$ being bounded in $L^{1}\left((0, T) ;\left(W^{2,2}(\Omega)\right)^{*}\right)$ and that upon another application of the Aubin-Lions lemma we obtain yet another subsequence along which (6.10) and $\nabla w_{\varepsilon} \rightarrow \nabla w$ in $L_{\text {loc }}^{2}\left(\bar{\Omega} \times[0, \infty) ; \mathbb{R}^{2}\right)$ hold. As $\rho>1$ we can than make use of Lemma 5.1 and Vitali's theorem to confirm that actually (6.13) is valid, whereas the weak convergences (6.11) and (6.12) result immediately from Lemmas 3.3 and 4.3, respectively. Finally, the strong convergence properties in (6.14) and (6.15) are a consequence of Vitali's theorem in conjunction with the equiintegrability properties contained in Lemmas 5.2 and 5.4, respectively.

The bounds presented in (6.16) follow from Lemma 3.2 in light of (6.1) and (6.6). To verify (6.17), we first note that according to (6.6) we can fix a null Set $N \subset(0, \infty)$ such that for all $t \in(0, \infty) \backslash N$ we have $v_{\varepsilon}(\cdot, t) \rightarrow v(\cdot, t)$ a.e. in $\Omega$ as $\varepsilon=\varepsilon_{j} \searrow 0$. Now, relying on Fatou's lemma and the estimate (4.2) from Lemma 4.1, we find by splitting $g$ into its positive and negative parts that

$$
\int_{\Omega} v(\cdot, t)+\int_{0}^{t} \int_{\Omega} g_{-}(v) \leq \liminf _{\varepsilon=\varepsilon_{j} \searrow 0}\left(\int_{\Omega} v_{\varepsilon}(\cdot, t)+\int_{0}^{t} \int_{\Omega} g_{-}\left(v_{\varepsilon}\right)\right)=\liminf _{\varepsilon=\varepsilon_{j} \searrow 0}\left(\int_{\Omega} v_{0}+\int_{0}^{t} \int_{\Omega} g_{+}\left(v_{\varepsilon}\right)\right)
$$

for all $t \in(0, \infty) \backslash N$. Since (1.6) implies that $g_{+}$is bounded on $(0, \infty)$, we can make use of the dominated convergence theorem and the continuity of $g_{+}$to pass to the limit on the right hand side and conclude (6.17). The nonnegativity properties are finally an immediate consequence of the nonnegativity of $u_{\varepsilon}, v_{\varepsilon}$ and $w_{\varepsilon}$ established in Lemma 3.1 combined with (6.1), (6.6) and (6.10), respectively. 


\section{Solution properties - Proof of Theorem 1.1}

With the limit objects $(u, v, w)$ provided by the previous proposition satisfying most of the requirements for a generalized solution in the sense of Definition 2.1, we are more or less left with checking that (2.1) - (2.3) are valid for these limit functions. Corresponding identities for the approximating systems (3.1) are easily obtainable from straightforward testing methods. It is, however, not clear yet that we may actually pass to the limit in each of the integrals involved. Let us first make sure that the equalities for $u$ and $w$ are satisfied.

\section{Lemma 7.1.}

Assume $\alpha>1+\sqrt{2}$ and $\beta>1$ and suppose that $\rho:=\min \{\alpha, \beta\}$ satisfies $\rho>\frac{\alpha+1}{\alpha-1}$. Let $\left(\varepsilon_{j}\right)_{j \in \mathbb{N}}$ and $(u, v, w)$ be as provided by Proposition 6.1. Then the identities (2.2) and (2.3) are satisfied for all $\varphi \in C_{0}^{\infty}(\bar{\Omega} \times[0, \infty))$.

Proof: Testing the first and third equation of (3.1) against an arbitrary test function $\varphi \in C_{0}^{\infty}(\bar{\Omega} \times[0, \infty))$ we obtain

$$
-\int_{0}^{\infty} \int_{\Omega} u_{\varepsilon} \varphi_{t}-\int_{\Omega} u_{0} \varphi(\cdot, 0)=-\int_{0}^{\infty} \int_{\Omega} \nabla u_{\varepsilon} \cdot \nabla \varphi+\int_{0}^{\infty} \int_{\Omega} u_{\varepsilon} \nabla w_{\varepsilon} \cdot \nabla \varphi+\int_{0}^{\infty} \int_{\Omega} f\left(u_{\varepsilon}\right) \varphi
$$

and

$$
-\int_{0}^{\infty} \int_{\Omega} w_{\varepsilon} \varphi_{t}-\int_{\Omega} w_{0} \varphi(\cdot, 0)=-\int_{0}^{\infty} \int_{\Omega} \nabla w_{\varepsilon} \cdot \nabla \varphi-\int_{0}^{\infty} \int_{\Omega} \frac{\left(u_{\varepsilon}+v_{\varepsilon}\right) w_{\varepsilon}}{1+\varepsilon\left(u_{\varepsilon}+v_{\varepsilon}\right) w_{\varepsilon}} \varphi-\mu \int_{0}^{\infty} \int_{\Omega} w_{\varepsilon} \varphi+\int_{0}^{\infty} \int_{\Omega} r \varphi,
$$

respectively, for all $\varepsilon \in(0,1)$. According to the convergence properties in $(6.1),(6.3),(6.15),(6.5)$, (6.10), (6.13) and (6.14) we may pass to the limit in each of the integrals above along the subsequence $\left(\varepsilon_{j}\right)_{j \in \mathbb{N}}$ provided by Proposition 6.1 and readily achieve (2.2) and (2.3).

The inequality for $v$ appearing in Definition 2.1 requires more work. In particular, in order to establish an identity corresponding to $(2.1)$ we will have to test the second equation of (3.1) against $\frac{\psi}{v_{\varepsilon}+1}$. The appearing integral $\int_{0}^{T} \int_{\Omega} \frac{v_{\varepsilon}}{v_{\varepsilon}+1}\left(\nabla \ln \left(v_{\varepsilon}+1\right) \cdot \nabla u_{\varepsilon}\right) \psi$ will then be the greatest adversary for our limit procedure, as it cannot be treated with the convergence results featured in Proposition 6.1. Due to the limited information attainable for $v_{\varepsilon}$ a better convergence property for $\nabla \ln \left(v_{\varepsilon}+1\right)$ appears to be far out of reach, necessitating that we establish a strong convergence for $\nabla u_{\varepsilon}$ instead. Thankfully, problems of this kind arise often in chemotaxis systems with generalized solution concepts (e.g. [40, 42, 5]) and can be treated with well-known results for Steklov averages and the convergence properties established in Proprosition 6.1.

Let us shortly recall that for $T>0, p \in[1, \infty], N \geq 1$ and $\psi \in L_{\text {loc }}^{p}\left(\bar{\Omega} \times(-1, T) ; \mathbb{R}^{N}\right)$ the Steklov averages $S_{h} \psi \in L^{p}\left(\Omega \times(0, T) ; \mathbb{R}^{N}\right), h \in(0,1)$, are defined by

$$
S_{h}(\psi)(x, t):=\frac{1}{h} \int_{t-h}^{t} \psi(x, s) \mathrm{d} s, \quad(x, t) \in \Omega \times(0, T)
$$

and satisfy $S_{h}(\psi) \rightarrow \psi$ in $L^{p}(\Omega \times(0, T))$ as $h \searrow 0$ if $p \in[1, \infty)$ and $S_{h}(\psi) \stackrel{\star}{\rightarrow} \psi$ in $L^{\infty}(\Omega \times(0, T))$ as $h \searrow 0$ if $p=\infty$ (cf. [40, Lemma 10.2]).

With this notation fixed, we can now enhance arguments akin to those presented in [40, Lemmas 8.1 and 8.2] and [42, Lemmas 5.1 and 5.2] to cover our current situation, with, in particular, the appearing source term $f\left(u_{\varepsilon}\right)$ requiring additional attention.

\section{Lemma 7.2.}

Assume $\alpha>1+\sqrt{2}$ and $\beta>1$ and suppose that $\rho:=\min \{\alpha, \beta\}$ satisfies $\rho>\frac{\alpha+1}{\alpha-1}$. Let $\left(\varepsilon_{j}\right)_{j \in \mathbb{N}}$ and $u$ be as provided by Proposition 6.1. Then for all $T>0$

$$
\nabla u_{\varepsilon} \rightarrow \nabla u \quad \text { in } L^{2}\left(\Omega \times(0, T) ; \mathbb{R}^{2}\right) \text { as } \varepsilon=\varepsilon_{j} \searrow 0 .
$$

Proof: Let $T>0$ be given. According to Proposition 6.1 we can choose $t_{0}>T$ such that $t_{0}$ is a Lebesgue point of the mapping $0<t \mapsto \int_{\Omega} u^{2}(\cdot, t)$ and that

$$
\int_{\Omega} u_{\varepsilon}^{2}\left(\cdot, t_{0}\right) \rightarrow \int_{\Omega} u^{2}\left(\cdot, t_{0}\right) \quad \text { as } \varepsilon=\varepsilon_{j} \searrow 0
$$


For $\delta \in(0,1)$ and the chosen $t_{0}>0$ we define the temporal cutoff functions

$$
\zeta_{\delta}(t) \equiv \zeta_{\delta}^{\left(t_{0}\right)}(t):= \begin{cases}1 & \text { if } t \in\left[0, t_{0}\right], \\ 1-\frac{t-t_{0}}{\delta} & \text { if } t \in\left(t_{0}, t_{0}+\delta\right), \\ 0 & \text { if } t \geq t_{0}+\delta\end{cases}
$$

which obviously satisfy

$$
\zeta_{\delta}^{\prime} \equiv \begin{cases}-\frac{1}{\delta} & \text { on }\left(t_{0}, t_{0}+\delta\right) \\ 0 & \text { on }\left(0, t_{0}\right) \cup\left(t_{0}+\delta, \infty\right)\end{cases}
$$

Moreover, we fix any sequence $\left(u_{0 k}\right)_{k \in \mathbb{N}} \subset C^{1}(\bar{\Omega})$ satisfying $u_{0 k} \rightarrow u_{0}$ in $L^{2}(\Omega)$ as $k \rightarrow \infty$ and accordingly let

$$
u_{k}(x, t):= \begin{cases}u(x, t) & \text { if } x \in \Omega \text { and } t>0 \\ u_{0 k}(x) & \text { if } x \in \Omega \text { and } t \leq 0\end{cases}
$$

With these preparations, for $h \in(0,1), \delta \in(0,1)$ and $k \in \mathbb{N}$ we construct the test functions

$$
\varphi(x, t):=\zeta_{\delta}(t) S_{h}\left(u_{k}\right)(x, t), \quad(x, t) \in \Omega \times(0, \infty),
$$

which satisfy $\varphi \in L^{\infty}\left((0, \infty) ; W^{1,2}(\Omega)\right)$ with $\varphi_{t} \in L^{2}(\Omega \times(0, \infty))$ and are compactly supported in $\bar{\Omega} \times\left[0, t_{0}+1\right]$. Making use of Lemma 7.1 and a completion argument we find that $\varphi$ is an admissible test function for (2.2), which entails that

$$
\begin{aligned}
& -\int_{0}^{\infty} \int_{\Omega} u(x, t) \zeta_{\delta}^{\prime}(t) S_{h}\left(u_{k}\right)(x, t) \mathrm{d} x \mathrm{~d} t \\
& -\int_{0}^{\infty} \int_{\Omega} u(x, t) \zeta_{\delta}(t) \frac{u_{k}(x, t)-u_{k}(x, t-h)}{h} \mathrm{~d} x \mathrm{~d} t-\int_{\Omega} u_{0}(x) u_{0 k}(x) \mathrm{d} x \\
& \quad=-\int_{0}^{\infty} \int_{\Omega} \nabla u \cdot \zeta_{\delta}(t) \nabla S_{h}\left(u_{k}\right)(x, t) \mathrm{d} x \mathrm{~d} t+\int_{0}^{\infty} \int_{\Omega} u(x, t) \nabla w(x, t) \cdot \zeta_{\delta}(t) \nabla S_{h}\left(u_{k}\right)(x, t) \mathrm{d} x \mathrm{~d} t \\
& \quad+\int_{0}^{\infty} \int_{\Omega} f(u(x, t)) \zeta_{\delta}(t) S_{h}\left(u_{k}\right)(x, t) \mathrm{d} x \mathrm{~d} t \quad \text { for all } h \in(0,1), k \in \mathbb{N} \text { and } \delta \in(0,1) .
\end{aligned}
$$

Here, we note that $\nabla u_{k} \in L^{2}\left(\Omega \times\left(-1, t_{0}+1\right) ; \mathbb{R}^{2}\right)$ and $u_{k} \in L^{\alpha+1}\left(\Omega \times\left(-1, t_{0}+1\right)\right)$ entails $\nabla S_{h}\left(u_{k}\right)=$ $S_{h}\left(\nabla u_{k}\right) \rightarrow \nabla u_{k}=\nabla u$ in $L^{2}\left(\Omega \times\left(0, t_{0}+1\right) ; \mathbb{R}^{2}\right)$ and $S_{h}\left(u_{k}\right) \rightarrow u$ in $L^{\alpha+1}\left(\Omega \times\left(0, t_{0}+1\right)\right)$ as $h \searrow 0$, which also obviously implies that $S_{h}\left(u_{k}\right) \rightarrow u$ in $L^{2}\left(\Omega \times\left(0, t_{0}+1\right)\right)$ due to $\alpha>1$. Since Proposition 6.1 ensures that $u$ belongs to $L^{2}\left(\Omega \times\left(0, t_{0}+1\right)\right)$, that $\nabla u$ as well as $u \nabla w$ belong to $L^{2}\left(\Omega \times\left(0, t_{0}+1\right) ; \mathbb{R}^{2}\right)$ and that $f(u)$ belongs to $L^{\frac{\alpha+1}{\alpha}}\left(\Omega \times\left(0, t_{0}+1\right)\right)$, we can take $h \searrow 0$ in each of the integrals on the right hand side and in the first integral on the left. To treat the remaining integral on the left we first employ Young's inequality to obtain that

$$
\begin{aligned}
& -\int_{0}^{\infty} \int_{\Omega} u(x, t) \zeta_{\delta}(t) \frac{u_{k}(x, t)-u_{k}(x, t-h)}{h} \\
= & -\frac{1}{h} \int_{0}^{\infty} \int_{\Omega} \zeta_{\delta}(t) u_{k}^{2}(x, t) \mathrm{d} x \mathrm{~d} t+\frac{1}{h} \int_{0}^{\infty} \int_{\Omega} \zeta_{\delta}(t) u_{k}(x, t) u_{k}(x, t-h) \mathrm{d} x \mathrm{~d} t \\
\leq & -\frac{1}{2 h} \int_{0}^{\infty} \int_{\Omega} \zeta_{\delta}(t) u_{k}^{2}(x, t) \mathrm{d} x \mathrm{~d} t+\frac{1}{2 h} \int_{0}^{\infty} \int_{\Omega} \zeta_{\delta}(t) u_{k}^{2}(x, t-h) \mathrm{d} x \mathrm{~d} t \\
= & -\frac{1}{2 h} \int_{0}^{\infty} \int_{\Omega} \zeta_{\delta}(t) u_{k}^{2}(x, t) \mathrm{d} x \mathrm{~d} t+\frac{1}{2 h} \int_{-h}^{\infty} \int_{\Omega} \zeta_{\delta}(s+h) u_{k}^{2}(x, s) \mathrm{d} x \mathrm{~d} s \\
= & \frac{1}{2} \int_{0}^{\infty} \int_{\Omega} u^{2}(x, s) \frac{\zeta_{\delta}(s+h)-\zeta_{\delta}(s)}{h} \mathrm{~d} x \mathrm{~d} s+\frac{1}{2} \int_{\Omega} u_{0 k}^{2}(x) \mathrm{d} x,
\end{aligned}
$$

where, by the dominated convergence theorem, we have

$$
\frac{1}{2} \int_{0}^{\infty} \int_{\Omega} u^{2}(x, s) \frac{\zeta_{\delta}(s+h)-\zeta_{\delta}(s)}{h} \mathrm{~d} x \mathrm{~d} s \rightarrow \frac{1}{2} \int_{0}^{\infty} \int_{\Omega} u^{2}(x, s) \zeta_{\delta}^{\prime}(s) \mathrm{d} x \mathrm{~d} s \quad \text { as } h \searrow 0 .
$$


Hence, passing to the limit $h \searrow 0$ in (7.3) we can make use of (7.2) to obtain that

$$
\begin{aligned}
& \frac{1}{2 \delta} \int_{t_{0}}^{t_{0}+\delta} \int_{\Omega} u^{2}(x, t) \mathrm{d} x \mathrm{~d} t+\frac{1}{2} \int_{\Omega} u_{0 k}^{2}(x) \mathrm{d} x-\int_{\Omega} u_{0}(x) u_{0 k}(x) \mathrm{d} x \\
& \geq- \int_{0}^{\infty} \int_{\Omega} \zeta_{\delta}(t)|\nabla u|^{2} \mathrm{~d} x \mathrm{~d} t+\int_{0}^{\infty} \int_{\Omega} \zeta_{\delta}(t) u(x, t)(\nabla w(x, t) \cdot \nabla u(x, t)) \mathrm{d} x \mathrm{~d} t \\
& \quad+\int_{0}^{\infty} \int_{\Omega} \zeta_{\delta}(t) f(u(x, t)) u(x, t) \mathrm{d} x \mathrm{~d} t \quad \text { for all } k \in \mathbb{N} \text { and } \delta \in(0,1) .
\end{aligned}
$$

Here, as $u_{0 k} \rightarrow u_{0}$ in $L^{2}(\Omega)$ as $k \rightarrow \infty$, we can first let $k \rightarrow \infty$ and then make use of the Lebesgue point property of $t_{0}$ and the dominated convergence theorem to let $\delta \searrow 0$ to find, upon appropriate reordering of the terms, that

$$
\int_{0}^{t_{0}} \int_{\Omega}|\nabla u|^{2} \geq-\frac{1}{2} \int_{\Omega} u^{2}\left(\cdot, t_{0}\right)+\frac{1}{2} \int_{\Omega} u_{0}^{2}+\int_{0}^{t_{0}} \int_{\Omega} u \nabla u \cdot \nabla w+\int_{0}^{t_{0}} \int_{\Omega} f(u) u .
$$

In order to link this with an suitable expression concerning the approximate systems (3.1) we make use of the strong convergence properties listed in (6.5) and (6.15) of Proposition 6.1, which together with (6.4) and (6.3), respectively, yield that

$$
\int_{0}^{t_{0}} \int_{\Omega} f\left(u_{\varepsilon}\right) u_{\varepsilon} \rightarrow \int_{0}^{t_{0}} \int_{\Omega} f(u) u \quad \text { and } \quad \int_{0}^{t_{0}} \int_{\Omega} u_{\varepsilon} \nabla u_{\varepsilon} \cdot \nabla w_{\varepsilon} \rightarrow \int_{0}^{t_{0}} \int_{\Omega} u \nabla u \cdot \nabla w \quad \text { as } \varepsilon=\varepsilon_{j} \searrow 0,
$$

and conclude from (7.4), and (7.1) and from testing the first equation of (3.1) against $u_{\varepsilon}$ that

$$
\begin{aligned}
\int_{0}^{t_{0}} \int_{\Omega}|\nabla u|^{2} & \geq \lim _{\varepsilon=\varepsilon_{j} \searrow 0}\left(-\frac{1}{2} \int_{\Omega} u_{\varepsilon}^{2}\left(\cdot, t_{0}\right)+\frac{1}{2} \int_{\Omega} u_{0}^{2}+\int_{0}^{t_{0}} \int_{\Omega} u_{\varepsilon} \nabla u_{\varepsilon} \cdot \nabla w_{\varepsilon}+\int_{0}^{t_{0}} \int_{\Omega} f\left(u_{\varepsilon}\right) u_{\varepsilon}\right) \\
& =\lim _{\varepsilon=\varepsilon_{j} \searrow 0} \int_{0}^{t_{0}} \int_{\Omega}\left|\nabla u_{\varepsilon}\right|^{2} .
\end{aligned}
$$

Due to $t_{0}>T$ and Proposition 6.1 saying that $\nabla u_{\varepsilon} \rightarrow \nabla u$ in $L^{2}\left(\Omega \times\left(0, t_{0}\right) ; \mathbb{R}^{2}\right)$ as $\varepsilon=\varepsilon_{j} \searrow 0$, we actually have $\nabla u_{\varepsilon} \rightarrow \nabla u$ in $L^{2}\left(\Omega \times(0, T) ; \mathbb{R}^{2}\right)$ as $\varepsilon=\varepsilon_{j} \searrow 0$, completing the proof.

With the strong convergence of $\nabla u_{\varepsilon}$ in $L^{2}\left(\Omega \times(0, T) ; \mathbb{R}^{2}\right)$ ensured by the previous lemma and the information contained in Proposition 6.1, we have now prepared all the major parts of the proof of Theorem 1.1 and can verify the solution properties of the limit objects in a straightforward fashion.

Proof of Theorem 1.1: Proposition 6.1 provides us with $(u, v, w)$ satisfying all the regularity and nonnegativity conditions required by Definition 2.1 as well as (2.4) and Lemma 7.1 verifies that $(2.2)$ and (2.3) hold, so that we are left with establishing (2.1). We first pick an arbitrary $\psi \in C_{0}^{\infty}(\bar{\Omega} \times[0, \infty))$ and then fix $T>0$ such that $\psi \equiv 0$ in $\Omega \times(T, \infty)$. Testing the second equation of (3.1) against $\frac{\psi}{v_{\varepsilon}+1}$ yields

$$
\begin{aligned}
-\int_{0}^{T} \int_{\Omega} \ln \left(v_{\varepsilon}+1\right) \psi_{t}- & \int_{\Omega} \ln \left(v_{0}+1\right) \psi(\cdot, 0) \\
=-\int_{0}^{T} \int_{\Omega} \nabla \ln \left(v_{\varepsilon}+1\right) \cdot \nabla \psi+\int_{0}^{T} \int_{\Omega}\left|\nabla \ln \left(v_{\varepsilon}+1\right)\right|^{2} \psi+\int_{0}^{T} \int_{\Omega} \frac{v_{\varepsilon}}{v_{\varepsilon}+1}\left(\nabla u_{\varepsilon} \cdot \nabla \psi\right) & \quad-\int_{0}^{T} \int_{\Omega} \frac{v_{\varepsilon}}{v_{\varepsilon}+1}\left(\nabla \ln \left(v_{\varepsilon}+1\right) \cdot \nabla u_{\varepsilon}\right) \psi+\int_{0}^{T} \int_{\Omega} \frac{g\left(v_{\varepsilon}\right)}{v_{\varepsilon}+1} \psi
\end{aligned}
$$

for all $\varepsilon \in(0,1)$. Now, with $\left(\varepsilon_{j}\right)_{j \in \mathbb{N}}$ provided by Proposition 6.1 we obtain from (6.7) and (6.9) that

$$
-\int_{0}^{T} \int_{\Omega} \ln \left(v_{\varepsilon}+1\right) \psi_{t} \rightarrow-\int_{0}^{T} \int_{\Omega} \ln (v+1) \psi_{t}, \quad-\int_{0}^{T} \int_{\Omega} \nabla \ln \left(v_{\varepsilon}+1\right) \cdot \nabla \psi \rightarrow-\int_{0}^{T} \int_{\Omega} \nabla \ln (v+1) \cdot \nabla \psi
$$

and

$$
\int_{0}^{T} \int_{\Omega} \frac{g\left(v_{\varepsilon}\right)}{v_{\varepsilon}+1} \psi \rightarrow \int_{0}^{T} \int_{\Omega} \frac{g(v)}{v+1} \psi \quad \text { as } \varepsilon=\varepsilon_{j} \searrow 0
$$


On the other hand, we can combine the boundedness of $\left|\frac{v_{\varepsilon}}{v_{\varepsilon}+1}\right|$ with the fact that $\frac{v_{\varepsilon}}{v_{\varepsilon}+1} \rightarrow \frac{v}{v+1}$ a.e. in $\Omega \times(0, T)$ as $\varepsilon=\varepsilon_{j} \searrow 0$ and the strong convergence from Lemma 7.2 to obtain that also

$$
\frac{v_{\varepsilon}}{v_{\varepsilon}+1} \nabla u_{\varepsilon} \rightarrow \frac{v}{v+1} \nabla u \quad \text { in } L^{2}\left(\Omega \times(0, T) ; \mathbb{R}^{2}\right)
$$

(see [40, Lemma 10.4]), so that

$$
\int_{0}^{T} \int_{\Omega} \frac{v_{\varepsilon}}{v_{\varepsilon}+1}\left(\nabla u_{\varepsilon} \cdot \nabla \psi\right) \rightarrow \int_{0}^{T} \int_{\Omega} \frac{v}{v+1}(\nabla u \cdot \nabla \psi)
$$

and

$$
-\int_{0}^{T} \int_{\Omega} \frac{v_{\varepsilon}}{v_{\varepsilon}+1}\left(\nabla \ln \left(v_{\varepsilon}+1\right) \cdot \nabla u_{\varepsilon}\right) \rightarrow-\int_{0}^{T} \int_{\Omega} \frac{v}{v+1}(\nabla \ln (v+1) \cdot \nabla u) \quad \text { as } \varepsilon=\varepsilon_{j} \searrow 0,
$$

where the second part is again due to (6.7). Finally, by the lower semicontinuity of the norm in $L^{2}(\Omega \times(0, T))$ with respect to weak convergence we obtain from (6.7) that

$$
\liminf _{\varepsilon=\varepsilon_{j} \searrow 0} \int_{0}^{T} \int_{\Omega}\left|\nabla \ln \left(v_{\varepsilon}+1\right)\right|^{2} \psi \geq \int_{0}^{T} \int_{\Omega}|\nabla \ln (v+1)|^{2} \psi .
$$

Hence, we can pass to the limit in all of the integrals appearing in (7.5), which in light of the fact that $\psi \equiv 0$ on $\Omega \times(T, \infty)$, entails

$$
\begin{aligned}
-\int_{0}^{\infty} \int_{\Omega} \ln (v+1) \psi_{t} & -\int_{\Omega} \ln \left(v_{0}+1\right) \psi \\
\geq-\int_{0}^{\infty} & \int_{\Omega} \ln (v+1) \cdot \nabla \psi+\int_{0}^{\infty} \int_{\Omega}|\nabla \ln (v+1)|^{2} \psi+\int_{0}^{\infty} \int_{\Omega} \frac{v}{v+1}(\nabla u \cdot \nabla \psi) \\
& -\int_{0}^{\infty} \int_{\Omega} \frac{v}{v+1}(\nabla \ln (v+1) \cdot \nabla \psi)+\int_{0}^{\infty} \int_{\Omega} \frac{g(v)}{v+1} \psi
\end{aligned}
$$

and thereby completes the proof.

\section{Conditional eventual regularity estimates}

In order to prove the eventual smoothing process postulated by Theorem 1.3 we will have to significantly improve our knowledge on the regularity of $u_{\varepsilon}$ and, especially, $v_{\varepsilon}$. In this section we will hence take an in-depth look at eventual regularity estimates which can be derived under the additional assumptions that at least beyond some waiting time $T>0$ we have certain (spatio-temporal) bounds at hand for $u_{\varepsilon}$, $v_{\varepsilon}$ and $w_{\varepsilon}$. We begin with a slightly refined version of Lemma 4.3.

\section{Corollary 8.1.}

Suppose that for some $q>1$ there are $T \geq 0$ and $K>0$ such that for any $\varepsilon \in(0,1)$ and all $t>T$ the solution $\left(u_{\varepsilon}, v_{\varepsilon}, w_{\varepsilon}\right)$ of $(3.1)$ satisfies

$$
\int_{t}^{t+1} \int_{\Omega} u_{\varepsilon}^{q}+\int_{t}^{t+1} \int_{\Omega} v_{\varepsilon}^{q} \leq K
$$

Then, there exists $C>0$ such that for any $\varepsilon \in(0,1)$ and all $t>T+1$

$$
\int_{t}^{t+1}\left\|w_{\varepsilon}\right\|_{W^{2, q}(\Omega)}^{q} \leq C
$$

Proof: This result is quite close to the one presented in Lemma 4.3. The main difference is the possibility of (8.1) only being valid after a waiting time $T \geq 0$ for which we pay by the fact that the bound on $w_{\varepsilon}$ only holds true for $t>T+1$. The proof, however, works along similar lines as Lemma 4.3. 
We only recall that upon picking $\tau>T$, letting $\xi:=\xi_{\tau}$ and employing the maximal Sobolev regularity estimates to the PDE corresponding to $\xi w_{\varepsilon}$ we find that there is some $C_{1}>0$ such that

$$
\int_{\tau}^{\tau+2}\left\|\xi w_{\varepsilon}\right\|_{W^{2, q}(\Omega)}^{q} \leq C_{1}+C_{1} \int_{\tau}^{\tau+2} \int_{\Omega} u_{\varepsilon}^{q}+C_{1} \int_{\tau}^{\tau+2} \int_{\Omega} v_{\varepsilon}^{q} \leq C_{1}+4 K C_{1},
$$

which, due to the arbitrary choice of $\tau>T$ and the fact that $\xi \equiv 1$ on $(\tau+1, \tau+2)$, implies that there is some $C_{2}>0$ such that

$$
\int_{t}^{t+1}\left\|w_{\varepsilon}\right\|_{W^{2, q}(\Omega)}^{q} \leq C_{2}
$$

holds for each $\varepsilon \in(0,1)$ and all $t>T+1$.

If we prescribe even stronger known eventual boundedness properties we can significantly improve the regularity of $u_{\varepsilon}$ and $v_{\varepsilon}$ in the next result. Due to its cyclic nature this result even provides a basis for an iterative argument we will later employ in Section 11.

Lemma 8.2.

Assume $\alpha>1+\sqrt{2}$ and $\beta>2$. Let $q>\max \left\{\beta-1, \frac{1}{\beta-2}\right\}$. If there are $\tau_{0}>0$ and $K_{0}>0$ such that

$$
\int_{t}^{t+1}\left\|w_{\varepsilon}\right\|_{W^{2, q}(\Omega)}^{q}+\int_{t}^{t+1} \int_{\Omega} v_{\varepsilon}^{q-\beta+2} \leq K_{0} \quad \text { for all } t>\tau_{0} \text { and } \varepsilon \in(0,1),
$$

and if for all $p \in[1, \infty)$ there are $\tau_{p}>0$ and $K_{p}>0$ such that

$$
\int_{\Omega} u_{\varepsilon}^{p}(\cdot, t) \leq K_{p} \quad \text { for all } t>\tau_{p} \text { and } \varepsilon \in(0,1),
$$

then for all $\theta \in(1, q)$ there exist $T_{\star}>0$ and $C>0$ such that for each $\varepsilon \in(0,1)$ and all $t>T_{\star}$

$$
\int_{t}^{t+1}\left\|u_{\varepsilon}\right\|_{W^{2, \theta}(\Omega)}^{\theta}+\int_{t}^{t+1} \int_{\Omega}\left|\nabla u_{\varepsilon}\right|^{2 \theta} \leq C
$$

and, moreover, there exists $C^{\prime}>0$ such that for each $\varepsilon \in(0,1)$ and all $t>T_{\star}$

$$
\int_{\Omega} v_{\varepsilon}^{q-\beta+2}(\cdot, t)+\int_{t}^{t+1} \int_{\Omega} v_{\varepsilon}^{q+1}+\int_{t}^{t+1} \int_{\Omega} v_{\varepsilon}^{q-\beta}\left|\nabla v_{\varepsilon}\right|^{2} \leq C^{\prime} \quad \text { and } \quad \int_{t}^{t+1}\left\|w_{\varepsilon}\right\|_{W^{2, q+1}(\Omega)}^{q+1} \leq C^{\prime} .
$$

Proof: We pick any $\theta \in(1, q)$ and let $a:=\max \left\{\alpha \theta, \frac{q \theta}{q-\theta}\right\}>1$. According to (8.2) and (8.3) for $\tau^{\prime}:=\max \left\{\tau_{0}, \tau_{a}\right\}>0$ and $K^{\prime}:=2 \max \left\{K_{0}, K_{a}\right\}>0$ we have

$$
\int_{t}^{t+1} \int_{\Omega}\left|\Delta w_{\varepsilon}\right|^{q}+\int_{t}^{t+1} \int_{\Omega} u_{\varepsilon}^{a} \leq K^{\prime} \quad \text { for any } \varepsilon \in(0,1) \text { and all } t>\tau^{\prime} .
$$

For $t_{0}>\tau^{\prime}$ we denote by $\xi:=\xi_{t_{0}}$ the cut-off function provided by Definition 4.2 and observe that $\xi u_{\varepsilon}$ solves the equation

$$
\begin{gathered}
\left(\xi u_{\varepsilon}\right)_{t}=\Delta\left(\xi u_{\varepsilon}\right)-\nabla\left(\xi u_{\varepsilon}\right) \cdot \nabla w_{\varepsilon}-\xi u_{\varepsilon} \Delta w_{\varepsilon}+\xi f\left(u_{\varepsilon}\right)+\xi^{\prime} u_{\varepsilon} \quad \text { in } \Omega \times\left(t_{0}, \infty\right) \\
\text { with }\left(\xi u_{\varepsilon}\right)\left(\cdot, t_{0}\right)=0 \text { in } \Omega \text { and } \nabla\left(\xi u_{\varepsilon}\right) \cdot \nu=0 \quad \text { on } \partial \Omega \times\left(t_{0}, \infty\right) .
\end{gathered}
$$

Now we once more rely on the maximal Sobolev regularity estimates $([13,11])$ and apply them to $(8.6)$. Thus, we find that for any $\theta \in(1, q)$ there is $C_{1}>0$ such that

$$
\begin{aligned}
\int_{t_{0}}^{t_{0}+2}\left\|\left(\xi u_{\varepsilon}\right)_{t}\right\|_{L^{\theta}(\Omega)}^{\theta}+\int_{t_{0}}^{t_{0}+2}\left\|\xi u_{\varepsilon}\right\|_{W^{2, \theta}(\Omega)}^{\theta} \leq & C_{1} \int_{t_{0}}^{t_{0}+2}\left\|\nabla\left(\xi u_{\varepsilon}\right) \cdot \nabla w_{\varepsilon}\right\|_{L^{\theta}(\Omega)}^{\theta}+C_{1} \int_{t_{0}}^{t_{0}+2}\left\|\xi u_{\varepsilon} \Delta w_{\varepsilon}\right\|_{L^{\theta}(\Omega)}^{\theta} \\
& +C_{1} \int_{t_{0}}^{t_{0}+2}\left\|\xi f\left(u_{\varepsilon}\right)\right\|_{L^{\theta}(\Omega)}^{\theta}+C_{1} \int_{t_{0}}^{t_{0}+2}\left\|\xi^{\prime} u_{\varepsilon}\right\|_{L^{\theta}(\Omega)}^{\theta}
\end{aligned}
$$


for all $\varepsilon \in(0,1)$. Making use of the fact that $\theta<q$, Young's inequality and (1.6) yield $C_{2}>0$ such that

$$
\begin{aligned}
& \int_{t_{0}}^{t_{0}+2}\left\|\xi u_{\varepsilon}\right\|_{W^{2, \theta}(\Omega)}^{\theta} \\
\leq & C_{1} \int_{t_{0}}^{t_{0}+2} \int_{\Omega}\left|\nabla\left(\xi u_{\varepsilon}\right)\right|^{\frac{2 q \theta}{2 q-\theta}}+C_{1} \int_{t_{0}}^{t_{0}+2} \int_{\Omega}\left|\nabla w_{\varepsilon}\right|^{2 q}+C_{1} \int_{t_{0}}^{t_{0}+2} \int_{\Omega}\left|\Delta w_{\varepsilon}\right|^{q}+C_{1} \int_{t_{0}}^{t_{0}+2} \int_{\Omega} u_{\varepsilon}^{\frac{q \theta}{q-\theta}} \\
& +C_{2} \int_{t_{0}}^{t_{0}+2} \int_{\Omega} u_{\varepsilon}^{\alpha \theta}+C_{2}+C_{1}\left\|\xi^{\prime}\right\|_{L^{\infty}(\mathbb{R})}^{\theta} \int_{t_{0}}^{t_{0}+2} \int_{\Omega} u_{\varepsilon}^{\theta} \text { for all } \varepsilon \in(0,1),
\end{aligned}
$$

where we also used that $\xi \leq 1$ on $\mathbb{R}$. To further estimate the terms on the right hand side, we note that in light of the Gagliardo-Nirenberg inequality (e.g. [9, Theorem I.9.3] combined with [9, Theorem I.19.1]) for any $1<s \leq \sigma$ we can find $C_{s, \sigma}>0$ such that

$$
\|\nabla \phi\|_{L^{2 s}(\Omega)}^{2 s} \leq C_{s, \sigma}\|\phi\|_{W^{2, \sigma}(\Omega)}^{s}\|\phi\|_{L^{\frac{s \sigma}{\sigma-s}}(\Omega)}^{s} \quad \text { for all } \phi \in W^{2, \sigma}(\Omega) .
$$

Since $\theta<q$ implies $\frac{q \theta}{2 q-\theta}<\theta$, we can employ (8.7) once for $\sigma=\theta$ and $s=\frac{q \theta}{2 q-\theta}$ and once for $\sigma=s=q$ to conclude the existence of $C_{3}>0$ satisfying

$$
\begin{aligned}
& \int_{t_{0}}^{t_{0}+2}\left\|\xi u_{\varepsilon}\right\|_{W^{2, \theta}(\Omega)}^{\theta} \\
& \leq C_{1} C_{3} \int_{t_{0}}^{t_{0}+2}\left\|\xi u_{\varepsilon}\right\|_{W^{2, \theta}(\Omega)}^{\frac{q \theta}{2 q-\theta}}\left\|\xi u_{\varepsilon}\right\|_{L^{\frac{q \theta}{q-\theta}}(\Omega)}^{\frac{q \theta}{2 q-\theta}}+C_{1} C_{3} \int_{t_{0}}^{t_{0}+2}\left\|w_{\varepsilon}\right\|_{W^{2, q}(\Omega)}^{q}\left\|w_{\varepsilon}\right\|_{L^{\infty}(\Omega)}^{q}+C_{1} \int_{t_{0}}^{t_{0}+2} \int_{\Omega}\left|\Delta w_{\varepsilon}\right|^{q} \\
& \quad+C_{1} \int_{t_{0}}^{t_{0}+2} \int_{\Omega} u_{\varepsilon}^{\frac{q \theta}{q-\theta}}+C_{2} \int_{t_{0}}^{t_{0}+2} \int_{\Omega} u_{\varepsilon}^{\alpha \theta}+C_{2}+C_{1}\left\|\xi^{\prime}\right\|_{L^{\infty}(\mathbb{R})}^{\theta} \int_{t_{0}}^{t_{0}+2} \int_{\Omega} u_{\varepsilon}^{\theta} \text { for all } \varepsilon \in(0,1) .
\end{aligned}
$$

Drawing once more on the fact that $\theta<q$ implies $\frac{q \theta}{2 q-\theta}<\theta$, we can rely on Young's inequality, Lemma 3.3 , and $\xi \leq 1$ on $\mathbb{R}$ to find $C_{4}>0$ such that

$$
\begin{aligned}
& \int_{t_{0}}^{t_{0}+2}\left\|\xi u_{\varepsilon}\right\|_{W^{2, \theta}(\Omega)}^{\theta} \\
& \leq \frac{1}{2} \int_{t_{0}}^{t_{0}+2}\left\|\xi u_{\varepsilon}\right\|_{W^{2, \theta}(\Omega)}^{\theta}+C_{4} \int_{t_{0}}^{t_{0}+2} \int_{\Omega} u_{\varepsilon}^{\frac{q \theta}{q-\theta}}+C_{1} C_{3} w_{*}^{q} \int_{t_{0}}^{t_{0}+2}\left\|w_{\varepsilon}\right\|_{W^{2, q}(\Omega)}^{q}+C_{1} \int_{t_{0}}^{t_{0}+2} \int_{\Omega}\left|\Delta w_{\varepsilon}\right|^{q} \\
& \quad+C_{1} \int_{t_{0}}^{t_{0}+2} \int_{\Omega} u_{\varepsilon}^{\frac{q \theta}{q-\theta}}+C_{2} \int_{t_{0}}^{t_{0}+2} \int_{\Omega} u_{\varepsilon}^{\alpha \theta}+C_{2}+C_{1}\left\|\xi^{\prime}\right\|_{L^{\infty}(\Omega)}^{\theta} \int_{t_{0}}^{t_{0}+2} \int_{\Omega} u_{\varepsilon}^{\theta} \text { for all } \varepsilon \in(0,1) .
\end{aligned}
$$

Due to $\alpha>1$ and $a=\max \left\{\alpha \theta, \frac{q \theta}{q-\theta}\right\}$, we can apply Young's inequality again, this time in the four integrals only containing $u_{\varepsilon}$, to obtain $C_{5}>0$ such that

$$
\frac{1}{2} \int_{t_{0}}^{t_{0}+2}\left\|\xi u_{\varepsilon}\right\|_{W^{2, \theta}(\Omega)}^{\theta} \leq C_{5} \int_{t_{0}}^{t_{0}+2} \int_{\Omega} u_{\varepsilon}^{a}+C_{1} C_{3} w_{*}^{q} \int_{t_{0}}^{t_{0}+2}\left\|w_{\varepsilon}\right\|_{W^{2, q}(\Omega)}^{q}+C_{1} \int_{t_{0}}^{t_{0}+2} \int_{\Omega}\left|\Delta w_{\varepsilon}\right|^{q}+C_{5}
$$

is valid for all $\varepsilon \in(0,1)$. In light of $(8.5)$, Lemma 3.3 combined with [9, Theorem I.19.1], the arbitrary choice of $t_{0}>\tau^{\prime}$ and the fact that $\xi \equiv 1$ on $\left(t_{0}+1, t_{0}+2\right)$ this entails the existence of $C_{6}>0$ such that

$$
\int_{t}^{t+1}\left\|u_{\varepsilon}\right\|_{W^{2, \theta}(\Omega)}^{\theta} \leq C_{6} \text { for each } \varepsilon \in(0,1) \text { and all } t>T_{1}:=\tau^{\prime}+1
$$

proving the first bound featured in (8.4). Next, for any $\theta \in(1, q)$ we pick $\theta^{*} \in(\theta, q)$ and find that, again in light of (8.7), there are $T_{2}>T_{1}$ and $C_{7}>0$ such that

$$
\int_{t}^{t+1} \int_{\Omega}\left|\nabla u_{\varepsilon}\right|^{2 \theta} \leq C_{\theta, \theta^{*}} \int_{t}^{t+1}\left\|u_{\varepsilon}\right\|_{W^{2, \theta^{*}}(\Omega)}^{\theta}\left\|u_{\varepsilon}\right\|_{L^{\frac{\theta \theta^{*}}{\theta^{*}-\theta}}(\Omega)}^{\theta} \leq C_{7} \quad \text { for each } \varepsilon \in(0,1) \text { and all } t>T_{2},
$$

due to (8.3), the choice $\theta^{*}<q$ and the first part of this lemma applied to $\theta^{*}$ instead of $\theta$. To verify the asserted boundedness for $v_{\varepsilon}$, we note that $q>\max \left\{\beta-1, \frac{1}{\beta-2}\right\}$ implies that $\lambda:=q-\beta+2>1$. Then, we turn to testing the second equation of (3.1) against $v_{\varepsilon}^{\lambda-1}$ and find upon integrating by parts that

$$
\frac{1}{\lambda} \frac{\mathrm{d}}{\mathrm{d} t} \int_{\Omega} v_{\varepsilon}^{\lambda}(\cdot, t)=-(\lambda-1) \int_{\Omega} v_{\varepsilon}^{\lambda-2}\left|\nabla v_{\varepsilon}\right|^{2}+(\lambda-1) \int_{\Omega} v_{\varepsilon}^{\lambda-1}\left(\nabla v_{\varepsilon} \cdot \nabla u_{\varepsilon}\right)+\int_{\Omega} v_{\varepsilon}^{\lambda-1} g\left(v_{\varepsilon}\right)
$$


holds on $(0, \infty)$. Therefore, drawing on the nonnegativity of $v_{\varepsilon},(1.6)$ and Young's inequality we obtain $C_{8}>0$ and $C_{9}>0$ such that for each $\varepsilon \in(0,1)$

$$
\begin{aligned}
\frac{1}{\lambda} \frac{\mathrm{d}}{\mathrm{d} t} \int_{\Omega} v_{\varepsilon}^{\lambda}(\cdot, t) & \leq-(\lambda-1) \int_{\Omega} v_{\varepsilon}^{\lambda-2}\left|\nabla v_{\varepsilon}\right|^{2}+(\lambda-1) \int_{\Omega} v_{\varepsilon}^{\lambda-1}\left(\nabla v_{\varepsilon} \cdot \nabla u_{\varepsilon}\right)-K_{g} \int_{\Omega} v_{\varepsilon}^{\lambda+\beta-1}+L_{g} \int_{\Omega} v_{\varepsilon}^{\lambda-1} \\
& \leq-\frac{\lambda-1}{2} \int_{\Omega} v_{\varepsilon}^{\lambda-2}\left|\nabla v_{\varepsilon}\right|^{2}-\frac{K_{g}}{2} \int_{\Omega} v_{\varepsilon}^{\lambda+\beta-1}+\frac{\lambda-1}{2} \int_{\Omega} v_{\varepsilon}^{\lambda}\left|\nabla u_{\varepsilon}\right|^{2}+C_{8} \\
& \leq-\frac{\lambda-1}{2} \int_{\Omega} v_{\varepsilon}^{\lambda-2}\left|\nabla v_{\varepsilon}\right|^{2}-\frac{K_{g}}{4} \int_{\Omega} v_{\varepsilon}^{\lambda+\beta-1}+C_{9} \int_{\Omega}\left|\nabla u_{\varepsilon}\right|^{\frac{2(\lambda+\beta-1)}{\beta-1}}+C_{8}
\end{aligned}
$$

is valid on $(0, \infty)$. Since (8.2) implies that for all $t>\tau_{0}+1$ and any $\varepsilon \in(0,1)$ there is some $T_{t}(\varepsilon) \in(t-1, t)$ such that

$$
\int_{\Omega} v_{\varepsilon}^{\lambda}\left(\cdot, T_{t}(\varepsilon)\right) \leq K_{0} \quad \text { for any } \varepsilon \in(0,1)
$$

we find from (8.8) that for any arbitrary $t>\tau_{0}+1$ and $\varepsilon \in(0,1)$

$$
\frac{1}{\lambda} \int_{\Omega} v_{\varepsilon}^{\lambda}(\cdot, t) \leq \frac{1}{\lambda} \int_{\Omega} v_{\varepsilon}^{\lambda}\left(\cdot, T_{t}(\varepsilon)\right)+C_{9} \int_{T_{t}(\varepsilon)}^{t} \int_{\Omega}\left|\nabla u_{\varepsilon}\right|^{\frac{2(\lambda+\beta-1)}{\beta-1}}+C_{8}\left(t-T_{t}(\varepsilon)\right),
$$

which due to $\frac{\lambda+\beta-1}{\beta-1}=\frac{q+1}{\beta-1}<q$, (8.3), the fact that $t-T_{t}(\varepsilon)<1$ for all $\varepsilon \in(0,1)$ and the arbitrary choice of $t>\tau_{0}+1$ yields $T_{3}>\max \left\{T_{2}, \tau_{0}+1\right\}$ and $C_{10}>0$, both independent of $\varepsilon \in(0,1)$, such that

$$
\frac{1}{\lambda} \int_{\Omega} v_{\varepsilon}^{q-\beta+2}(\cdot, t) \leq \frac{K_{0}}{\lambda}+C_{10} \text { for each } \varepsilon \in(0,1) \text { and all } t>T_{3} .
$$

With this, we return to (8.8) and find $C_{11}>0$ such that

$$
\begin{gathered}
\frac{K_{g}}{4} \int_{t}^{t+1} \int_{\Omega} v_{\varepsilon}^{q+1}+\frac{q-\beta+1}{2} \int_{t}^{t+1} \int_{\Omega} v_{\varepsilon}^{q-\beta}\left|\nabla v_{\varepsilon}\right|^{2} \\
\leq \frac{1}{q-\beta+2} \int_{\Omega} v_{\varepsilon}^{q-\beta+2}(\cdot, t)+C_{9} \int_{t}^{t+1} \int_{\Omega}\left|\nabla u_{\varepsilon}\right|^{\frac{2(q+1)}{\beta-1}}+C_{8} \leq C_{11} \quad \text { for each } \varepsilon \in(0,1) \text { and all } t>T_{3},
\end{gathered}
$$

as claimed. Finally, we observe that the bound on $\int_{t}^{t+1} \int_{\Omega} v_{\varepsilon}^{q+1}$ for all $t>T_{3}$, when combined with the fact that by (8.3) there is some $T_{4} \geq T_{3}$ such that $u_{\varepsilon}$ is bounded in $L^{q+1}(\Omega)$ for all $t>T_{4}$ and an application of Corollary 8.1, immediately yields $C_{11}>0$ such that

$$
\int_{t}^{t+1}\left\|w_{\varepsilon}\right\|_{W^{2, q+1}(\Omega)}^{q+1} \leq C_{11} \quad \text { for each } \varepsilon \in(0,1) \text { and all } t>T_{4},
$$

which completes the proof.

\section{Decay properties of $w_{\varepsilon}$}

While at least some of the bounds appearing in the conditions of Lemma 8.2 are similar to those already established in previous sections, we are still missing the required eventual $L^{q}$ regularity of $u_{\varepsilon}$ for arbitrary large $q \geq 1$. Comparing with similar chemotaxis systems where the eventual smoothness of solutions could be observed, one approach to this boundedness consists of a combination of a decay property of the nutrient density and a differential inequality for the quantity $\int_{\Omega} \frac{u_{\varepsilon}^{q}}{\left(2 \delta-w_{\varepsilon}\right)^{\theta}}$ with small $\delta>0$ and suitably chosen $\theta, T>0$ (see e.g. [20,39]). To make use of this approach, however, we first have to ensure that $w_{\varepsilon}$ indeed does decay below any given threshold, provided the waiting time is large enough. As an initial step we will ensure that at least $\left\|w_{\varepsilon}\right\|_{L^{1}(\Omega \times(t, t+1))}$ does decay past any positive threshold.

\section{Lemma 9.1.}

Assume $\mu>0, \alpha>1+\sqrt{2}$ and $\beta>1$ and suppose that $\rho:=\min \{\alpha, \beta\}$ satisfies $\rho>\frac{\alpha+1}{\alpha-1}$ and that $r \in C^{1}(\bar{\Omega} \times[0, \infty)) \cap L^{\infty}(\Omega \times(0, \infty)) \cap L^{1}\left((0, \infty) ; L^{\infty}(\Omega)\right)$ fulfills $(1.10)$. Then for any $\delta>0$ there exists $T>0$ such that for all $\varepsilon \in(0,1)$ the solution $\left(u_{\varepsilon}, v_{\varepsilon}, w_{\varepsilon}\right)$ of $(3.1)$ satisfies

$$
\int_{T}^{\infty} \int_{\Omega} \frac{\left(u_{\varepsilon}+v_{\varepsilon}\right) w_{\varepsilon}}{1+\varepsilon\left(u_{\varepsilon}+v_{\varepsilon}\right) w_{\varepsilon}}+\int_{T}^{\infty} \int_{\Omega} w_{\varepsilon}<\delta
$$


Proof: We infer from the integrating third equation of (3.1) and the divergence theorem that

$$
\frac{\mathrm{d}}{\mathrm{d} t} \int_{\Omega} w_{\varepsilon}(\cdot, t)+\int_{\Omega}\left(\frac{\left(u_{\varepsilon}+v_{\varepsilon}\right) w_{\varepsilon}}{1+\varepsilon\left(u_{\varepsilon}+v_{\varepsilon}\right) w_{\varepsilon}}\right)(\cdot, t)+\mu \int_{\Omega} w_{\varepsilon}(\cdot, t) \leq \int_{\Omega} r(\cdot, t)
$$

for all $t>0$. Hence, due to the nonnegativity of $w_{\varepsilon}$ on $\Omega \times(0, \infty)$ and $(1.10)$,

$$
\int_{0}^{t} \int_{\Omega} \frac{\left(u_{\varepsilon}+v_{\varepsilon}\right) w_{\varepsilon}}{1+\varepsilon\left(u_{\varepsilon}+v_{\varepsilon}\right) w_{\varepsilon}}+\mu \int_{0}^{t} \int_{\Omega} w_{\varepsilon} \leq \int_{\Omega} w_{0}+r_{* *}
$$

is valid for all $t>0$, from which we can readily conclude the desired outcome.

Following the ideas of $[42$, Lemma 6.7$]$ we can now verify that also $\left\|w_{\varepsilon}(\cdot, t)\right\|_{L^{\infty}(\Omega)}$ decays to zero.

\section{Lemma 9.2.}

Assume $\mu>0, \alpha>1+\sqrt{2}$ and $\beta>1$ and suppose that $\rho:=\min \{\alpha, \beta\}$ satisfies $\rho>\frac{\alpha+1}{\alpha-1}$ and that $r \in C^{1}(\bar{\Omega} \times[0, \infty)) \cap L^{\infty}(\Omega \times(0, \infty)) \cap L^{1}\left((0, \infty) ; L^{\infty}(\Omega)\right)$ fulfills $(1.10)$. Then for any $\delta>0$ there is $T>0$ such that for any $t>T$ and all $\varepsilon \in(0,1)$ the solution $\left(u_{\varepsilon}, v_{\varepsilon}, w_{\varepsilon}\right)$ of $(3.1)$ satisfies

$$
\left\|w_{\varepsilon}(\cdot, t)\right\|_{L^{\infty}(\Omega)}<\delta
$$

Proof: According to well-known estimates for the Neumann heat semigroup (e.g. [36, Lemma 1.3]) we can find $C_{1}>0$ such that

$$
\left\|e^{\sigma \Delta} \varphi\right\|_{L^{\infty}(\Omega)} \leq C_{1} \sigma^{-1}\|\varphi\|_{L^{1}(\Omega)} \quad \text { for all } \sigma \in(0,1) \text { and } \varphi \in C^{0}(\bar{\Omega}) \text { with } \bar{\varphi}=0,
$$

where $\bar{\varphi}:=\frac{1}{|\Omega|} \int_{\Omega} \varphi$. Now, given any arbitrary $\delta>0$ we note that by Lemma 9.1 there is some $T_{1}>1$ such that for all $t>T_{1}$ and any $\varepsilon \in(0,1)$

$$
2 C_{1} \int_{t-1}^{t-\frac{1}{2}}\left\|w_{\varepsilon}(\cdot, s)\right\|_{L^{1}(\Omega)} \mathrm{d} s<\frac{\delta}{4},
$$

and that in light of $(1.10)$ we can pick $T_{2}>1$ such that

$$
\int_{t-1}^{t}\|r(\cdot, s)\|_{L^{\infty}(\Omega)} \mathrm{d} s<\frac{\delta}{2} \quad \text { for all } t>T_{2} .
$$

Letting $T_{0}:=\max \left\{T_{1}, T_{2}\right\}$ we fix an arbitrary $t>T_{0}$ and note that from (9.1) we can conclude that there is some $t_{0} \in\left(t-1, t-\frac{1}{2}\right)$ satisfying

$$
2 C_{1}\left\|w_{\varepsilon}\left(\cdot, t_{0}\right)\right\|_{L^{1}(\Omega)}<\frac{\delta}{2} \text { for all } \varepsilon \in(0,1) .
$$

With this we now observe that the comparison principle implies that

$$
\begin{aligned}
w_{\varepsilon}(\cdot, t) & =e^{\left(t-t_{0}\right) \Delta} w_{\varepsilon}\left(\cdot, t_{0}\right)-\int_{t_{0}}^{t} e^{(t-s) \Delta}\left(\frac{\left(u_{\varepsilon}+v_{\varepsilon}\right) w_{\varepsilon}}{1+\varepsilon\left(u_{\varepsilon}+v_{\varepsilon}\right) w_{\varepsilon}}(\cdot, s)+\mu w_{\varepsilon}(\cdot, s)\right) \mathrm{d} s+\int_{t_{0}}^{t} e^{(t-s) \Delta} r(\cdot, s) \mathrm{d} s \\
& \leq e^{\left(t-t_{0}\right) \Delta}\left(w_{\varepsilon}\left(\cdot, t_{0}\right)-\overline{w_{\varepsilon}\left(\cdot, t_{0}\right)}\right)+\overline{w_{\varepsilon}\left(\cdot, t_{0}\right)}+\int_{t_{0}}^{t}\|r(\cdot, s)\|_{L^{\infty}(\Omega)} \mathrm{d} s \quad \text { in } \Omega,
\end{aligned}
$$

and that hence

$$
\begin{aligned}
\left\|w_{\varepsilon}(\cdot, t)\right\|_{L^{\infty}(\Omega)} & \leq C_{1}\left(t-t_{0}\right)^{-1}\left\|w_{\varepsilon}\left(\cdot, t_{0}\right)\right\|_{L^{1}(\Omega)}+\left\|\overline{w_{\varepsilon}\left(\cdot, t_{0}\right)}\right\|_{L^{\infty}(\Omega)}+\int_{t_{0}}^{t}\|r(\cdot, s)\|_{L^{\infty}(\Omega)} \mathrm{d} s \\
& \leq\left(2 C_{1}+\frac{1}{|\Omega|}\right)\left\|w_{\varepsilon}\left(\cdot, t_{0}\right)\right\|_{L^{1}(\Omega)}+\int_{t_{0}}^{t_{0}+1}\|r(\cdot, s)\|_{L^{\infty}(\Omega)} \mathrm{d} s<\frac{\delta}{2}+\frac{\delta}{2}=\delta
\end{aligned}
$$

for all $\varepsilon \in(0,1)$, completing the proof. 


\section{Eventual $L^{p}$ regularity of $u_{\varepsilon}$}

With the decay property of $w_{\varepsilon}$ at hand, we can now turn to tracking the time-evolution of $\int_{\Omega} \frac{u_{\varepsilon}^{q}}{\left(2 \delta-w_{\varepsilon}\right)^{\theta}}$. Functionals of this type have successfully been utilized in e.g. [20, Lemma 3.5] and [39, Lemma 5.1] in similar chemotaxis system.

\section{Lemma 10.1.}

Let $q>1, \theta>0, \delta \in\left(0, \frac{1}{2 q}\right)$ and $T>0$. Then there is $C>0$ such that whenever the solution $\left(u_{\varepsilon}, v_{\varepsilon}, w_{\varepsilon}\right)$ of (3.1) fulfills

$$
\left\|w_{\varepsilon}(\cdot, t)\right\|_{L^{\infty}(\Omega)} \leq \delta \quad \text { for all } \varepsilon \in(0,1) \text { and } t>T
$$

then the inequality

$$
\begin{aligned}
\frac{\mathrm{d}}{\mathrm{d} t} \int_{\Omega} \frac{u_{\varepsilon}^{q}}{\left(2 \delta-w_{\varepsilon}\right)^{\theta}} & +q K_{f} \int_{\Omega} \frac{u_{\varepsilon}^{q+\alpha-1}}{\left(2 \delta-w_{\varepsilon}\right)^{\theta}} \\
& \leq\left[\frac{(2 q \theta+2 q(q-1) \delta)^{2}}{4(\theta(\theta+1)-2 q \theta \delta)}-q(q-1)\right] \int_{\Omega} \frac{u_{\varepsilon}^{q-2}\left|\nabla u_{\varepsilon}\right|^{2}}{\left(2 \delta-w_{\varepsilon}\right)^{\theta}}+C \int_{\Omega} \frac{u_{\varepsilon}^{q}}{\left(2 \delta-w_{\varepsilon}\right)^{\theta}}+C
\end{aligned}
$$

holds for all $t>T$ and $\varepsilon \in(0,1)$.

Proof: Straightforward calculations utilizing integration by parts shows

$$
\begin{aligned}
& \frac{\mathrm{d}}{\mathrm{d} t} \int_{\Omega} \frac{u_{\varepsilon}^{q}}{\left(2 \delta-w_{\varepsilon} \theta^{\theta}\right.} \\
= & q \int_{\Omega} \frac{u_{\varepsilon}^{q-1}\left(\Delta u_{\varepsilon}-\nabla \cdot\left(u_{\varepsilon} \nabla w_{\varepsilon}\right)+f\left(u_{\varepsilon}\right)\right)}{\left(2 \delta-w_{\varepsilon}\right)^{\theta}}+\theta \int_{\Omega} \frac{u_{\varepsilon}^{q}}{\left(2 \delta-w_{\varepsilon}\right)^{\theta+1}}\left(\Delta w_{\varepsilon}-\frac{\left(u_{\varepsilon}+v_{\varepsilon}\right) w_{\varepsilon}}{1+\varepsilon\left(u_{\varepsilon}+v_{\varepsilon}\right) w_{\varepsilon}}-\mu w_{\varepsilon}+r\right) \\
= & -q(q-1) \int_{\Omega} \frac{u_{\varepsilon}^{q-2}\left|\nabla u_{\varepsilon}\right|^{2}}{\left(2 \delta-w_{\varepsilon}\right)^{\theta}}-2 q \theta \int_{\Omega} \frac{u_{\varepsilon}^{q-1}}{\left(2 \delta-w_{\varepsilon}\right)^{\theta+1}}\left(\nabla u_{\varepsilon} \cdot \nabla w_{\varepsilon}\right)+q(q-1) \int_{\Omega} \frac{u_{\varepsilon}^{q-1}}{\left(2 \delta-w_{\varepsilon} \theta^{\theta}\right.}\left(\nabla u_{\varepsilon} \cdot \nabla w_{\varepsilon}\right) \\
& \quad+q \theta \int_{\Omega} \frac{u_{\varepsilon}^{q}\left|\nabla w_{\varepsilon}\right|^{2}}{\left(2 \delta-w_{\varepsilon}\right)^{\theta+1}}+q \int_{\Omega} \frac{u_{\varepsilon}^{q-1} f\left(u_{\varepsilon}\right)}{\left(2 \delta-w_{\varepsilon}\right)^{\theta}}-\theta(\theta+1) \int_{\Omega} \frac{u_{\varepsilon}^{q}\left|\nabla w_{\varepsilon}\right|^{2}}{\left(2 \delta-w_{\varepsilon}\right)^{\theta+2}} \\
& \quad-\theta \int_{\Omega} \frac{\left(u_{\varepsilon}+v_{\varepsilon}\right) w_{\varepsilon} u_{\varepsilon}^{q}}{\left(1+\varepsilon\left(u_{\varepsilon}+v_{\varepsilon}\right) w_{\varepsilon}\right)\left(2 \delta-w_{\varepsilon}\right)^{\theta+1}}-\mu \theta \int_{\Omega} \frac{u_{\varepsilon}^{q} w_{\varepsilon}}{\left(2 \delta-w_{\varepsilon}\right)^{\theta+1}}+\theta \int_{\Omega} \frac{r u_{\varepsilon}^{q}}{\left(2 \delta-w_{\varepsilon}\right)^{\theta+1}}
\end{aligned}
$$

for all $t>T$. Therefore, relying on (1.6), (1.7), the nonegativity of $u_{\varepsilon}, v_{\varepsilon}, w_{\varepsilon}$ and $\mu$, as well as on the fact that $\frac{1}{2 \delta} \leq \frac{1}{2 \delta-w_{\varepsilon}} \leq \frac{1}{\delta}$ we can estimate

$$
\begin{aligned}
& \frac{\mathrm{d}}{\mathrm{d} t} \int_{\Omega} \frac{u_{\varepsilon}^{q}}{\left(2 \delta-w_{\varepsilon}\right)^{\theta}} \\
\leq & -q(q-1) \int_{\Omega} \frac{u_{\varepsilon}^{q-2}\left|\nabla u_{\varepsilon}\right|^{2}}{\left(2 \delta-w_{\varepsilon}\right)^{\theta}}+(2 q \theta+2 q(q-1) \delta) \int_{\Omega} \frac{u_{\varepsilon}^{q-1}\left|\nabla u_{\varepsilon}\right|\left|\nabla w_{\varepsilon}\right|}{\left(2 \delta-w_{\varepsilon}\right)^{\theta+1}}-(\theta(\theta+1)-2 q \theta \delta) \int_{\Omega} \frac{u_{\varepsilon}^{q}\left|\nabla w_{\varepsilon}\right|^{2}}{\left(2 \delta-w_{\varepsilon}\right)^{\theta+2}} \\
& -q K_{f} \int_{\Omega} \frac{u_{\varepsilon}^{q+\alpha-1}}{\left(2 \delta-w_{\varepsilon}\right)^{\theta}}+q L_{f} \int_{\Omega} \frac{u_{\varepsilon}^{q-1}}{\left(2 \delta-w_{\varepsilon}\right)^{\theta}}+\frac{\theta r_{*}}{\delta} \int_{\Omega} \frac{u_{\varepsilon}^{q}}{\left(2 \delta-w_{\varepsilon}\right)^{\theta}}
\end{aligned}
$$

for all $t>T$. To treat some of the terms further, we apply Young's inequality to the second term on the right, yielding

$$
\int_{\Omega} \frac{u_{\varepsilon}^{q-1}\left|\nabla u_{\varepsilon}\right|\left|\nabla w_{\varepsilon}\right|}{\left(2 \delta-w_{\varepsilon}\right)^{\theta+1}} \leq \frac{(2 q \theta+2 q(q-1) \delta)}{4(\theta(\theta+1)-2 q \theta \delta)} \int_{\Omega} \frac{u_{\varepsilon}^{q-2}\left|\nabla u_{\varepsilon}\right|^{2}}{\left(2 \delta-w_{\varepsilon}\right)^{\theta}}+\frac{(\theta(\theta+1)-2 q \theta \delta)}{(2 q \theta+2 q(q-1) \delta)} \int_{\Omega} \frac{u_{\varepsilon}^{q}\left|\nabla w_{\varepsilon}\right|^{2}}{\left(2 \delta-w_{\varepsilon}\right)^{\theta+2}}
$$

and to the second to last term on the right, providing some $C_{1}>0$ such that

$$
q L_{f} \int_{\Omega} \frac{u_{\varepsilon}^{q-1}}{\left(2 \delta-w_{\varepsilon}\right)^{\theta}} \leq \int_{\Omega} \frac{u_{\varepsilon}^{q}}{\left(2 \delta-w_{\varepsilon}\right)^{\theta}}+C_{1} \int_{\Omega} \frac{1}{\left(2 \delta-w_{\varepsilon}\right)^{\theta}} \leq \int_{\Omega} \frac{u_{\varepsilon}^{q}}{\left(2 \delta-w_{\varepsilon}\right)^{\theta}}+\frac{C_{1}}{\delta^{\theta}} \quad \text { for all } t>T .
$$

Plugging these two estimates into (10.1) finally completes the proof. 
In order to get rid of the last gradient term appearing in the differential inequality of the previous lemma, we will rely on the decay property of $w_{\varepsilon}$ to choose $\delta$ sufficiently small. The choice of $\delta$ is quite similar to the reasoning found in [20, Lemma 3.5], however, due to the more general form of $f$, here we will require some additional information to make successful use of the resulting differential inequality.

\section{Lemma 10.2.}

Assume $\mu>0, \alpha>1+\sqrt{2}$ and $\beta>1$ and suppose that $\rho:=\min \{\alpha, \beta\}$ satisfies $\rho>\frac{\alpha+1}{\alpha-1}$ and that $r \in C^{1}(\bar{\Omega} \times[0, \infty)) \cap L^{\infty}(\Omega \times(0, \infty)) \cap L^{1}\left((0, \infty) ; L^{\infty}(\Omega)\right)$ fulfills $(1.10)$. Then for all $q>1$ there exist $T>0$ and $C>0$ such that for each $\varepsilon \in(0,1)$ and all $t>T$ the solution $\left(u_{\varepsilon}, v_{\varepsilon}, w_{\varepsilon}\right)$ of $(3.1)$ fulfills

$$
\int_{\Omega} u_{\varepsilon}^{q}(\cdot, t) \leq C
$$

Proof: Given $q>1$ we fix a small $\theta>0$ satisfying

$$
4\left(q+2 q(q-1)+q(q-1)^{2}\right) \theta<2(q-1)
$$

and then pick $0<\delta<\min \left\{1, \theta, \frac{1}{4 q}\right\}$. Since $\delta<\frac{1}{4 q}$ implies $1<2(\theta+1-2 q \delta)$ and $\delta<\theta$ entails $\frac{\delta}{\theta}<1$, we conclude from (10.2) that

$$
4\left(q+2 q(q-1) \frac{\delta}{\theta}+q(q-1)^{2} \frac{\delta^{2}}{\theta^{2}}\right) \theta<4(q-1)(\theta+1-2 q \delta),
$$

which, upon simple rearrangement, may also be expressed as

$$
\frac{(2 q \theta+2 q(q-1) \delta)^{2}}{4(\theta(\theta+1)-2 q \theta \delta)}<q(q-1) .
$$

With these parameters fixed, we then employ Lemma 9.2 to find $T>0$ such that

$$
\left\|w_{\varepsilon}(\cdot, t)\right\|_{L^{\infty}(\Omega)}<\delta \quad \text { for all } \varepsilon \in(0,1) \text { and } t>T
$$

and conclude from Lemma 10.1 and (10.3) that there is some $C_{1}>0$ such that

$$
\frac{\mathrm{d}}{\mathrm{d} t} \int_{\Omega} \frac{u_{\varepsilon}^{q}}{\left(2 \delta-w_{\varepsilon}\right)^{\theta}}+q K_{f} \int_{\Omega} \frac{u_{\varepsilon}^{q+\alpha-1}}{\left(2 \delta-w_{\varepsilon}\right)^{\theta}} \leq C_{1} \int_{\Omega} \frac{u_{\varepsilon}^{q}}{\left(2 \delta-w_{\varepsilon}\right)^{\theta}}+C_{1} \quad \text { for all } \varepsilon \in(0,1) \text { and } t>T .
$$

Here, Hölder's inequality and Young's inequality entail the existence of $C_{2}>0$ such that

$$
2 C_{1} \int_{\Omega} \frac{u_{\varepsilon}^{q}}{\left(2 \delta-w_{\varepsilon}\right)^{\theta}} \leq 2 C_{1}\left(\frac{|\Omega|}{\delta^{\theta}}\right)^{\frac{\alpha-1}{q+\alpha-1}}\left(\int_{\Omega} \frac{u_{\varepsilon}^{q+\alpha-1}}{\left(2 \delta-w_{\varepsilon}\right)^{\theta}}\right)^{\frac{q}{q+\alpha-1}} \leq q K_{f} \int_{\Omega} \frac{u_{\varepsilon}^{q+\alpha-1}}{\left(2 \delta-w_{\varepsilon}\right)^{\theta}}+C_{2}
$$

for all $\varepsilon \in(0,1)$ and $t>T$, so that upon letting $y_{\varepsilon}(t):=\int_{\Omega} \frac{u_{\varepsilon}^{q}(\cdot, t)}{\left(2 \delta-w_{\varepsilon}(\cdot, t)\right)^{\theta}}, t>T$, we find that $y_{\varepsilon}$ satisfies

$$
y_{\varepsilon}^{\prime}+C_{1} y_{\varepsilon} \leq C_{1}+C_{2}=: C_{3} \text { for all } \varepsilon \in(0,1) \text { and } t>T .
$$

To exploit the information contained in this differential inequality successfully, we note that according to Corollary 4.7 there is some $C_{4}>0$ such that $\int_{T}^{T+1} \int_{\Omega}\left|\nabla u_{\varepsilon}\right|^{2} \leq C_{4}$ and that hence for each $\varepsilon \in(0,1)$ we can find $t_{\varepsilon} \in(T, T+1)$ such that $\int_{\Omega}\left|\nabla u_{\varepsilon}\left(\cdot, t_{\varepsilon}\right)\right|^{2} \leq C_{4}$. In view of the embedding $W^{1,2}(\Omega) \hookrightarrow L^{q}(\Omega)$ this also readily implies that there is some $C_{5}>0$ such that

$$
\int_{\Omega} u_{\varepsilon}^{q}\left(\cdot, t_{\varepsilon}\right) \leq C_{5} \quad \text { for all } \varepsilon \in(0,1)
$$

From a combination with the ODE comparison argument from Lemma 4.5 applied to (10.4) we can therefore conclude the existence of $C_{6}>0$ such that

$$
y_{\varepsilon}(t) \leq y_{\varepsilon}\left(t_{\varepsilon}\right)+\frac{C_{3}}{1-e^{-C_{1}}} \leq C_{6} \quad \text { for all } \varepsilon \in(0,1) \text { and all } t>T+2>t_{\varepsilon}+1 .
$$

Due to $\left(2 \delta-w_{\varepsilon}\right)^{\theta} \leq(2 \delta)^{\theta}$ on $\Omega \times(T, \infty)$ we can finally establish that for all $\varepsilon \in(0,1)$

$$
\int_{\Omega} u_{\varepsilon}^{q}=\int_{\Omega}\left(2 \delta-w_{\varepsilon}\right)^{\theta} \frac{u_{\varepsilon}^{q}}{\left(2 \delta-w_{\varepsilon}\right)^{\theta}} \leq(2 \delta)^{\theta} \int_{\Omega} \frac{u_{\varepsilon}^{q}}{\left(2 \delta-w_{\varepsilon}\right)^{\theta}}=(2 \delta)^{\theta} y_{\varepsilon}(t) \leq(2 \delta)^{\theta} C_{6} \quad \text { on }(T+2, \infty),
$$

completing the proof. 


\section{Eventual smoothness - Proof of Theorem 1.3}

With the eventual $L^{q}$ bounds of $u_{\varepsilon}$ for any $q>1$ from the previous section at hand, we can now return to our conditional estimates of Section 8 to lift the regularity information on the solution in an iterative procedure even higher, finally concluding in an eventual Hölder estimates for both $u_{\varepsilon}$ and $v_{\varepsilon}$. For this, however, we have to ensure that the spatio-temporal bound on $v_{\varepsilon}$ in Lemma 4.1 is sufficiently strong to work as a starting point for this procedure, which is the essential reason why we will have to restrict ourselves to values of $\beta>1+\sqrt{2}$.

\section{Lemma 11.1.}

Assume $\mu>0, \alpha>1+\sqrt{2}$ and $\beta>1+\sqrt{2}$ and that $r \in C^{1}(\bar{\Omega} \times[0, \infty)) \cap L^{\infty}(\Omega \times(0, \infty)) \cap$ $L^{1}\left((0, \infty) ; L^{\infty}(\Omega)\right)$ fulfills (1.10). Then for any $p \in(1, \infty)$ there are $T>0$ and $C>0$ such that for each $\varepsilon \in(0,1)$ and all $t>T$ the solution $\left(u_{\varepsilon}, v_{\varepsilon}, w_{\varepsilon}\right)$ of $(3.1)$ satisfies

$$
\int_{\Omega} v_{\varepsilon}^{p}(\cdot, t)+\int_{t}^{t+1}\left\|u_{\varepsilon}\right\|_{W^{2, p}(\Omega)}^{p}+\int_{t}^{t+1}\left\|w_{\varepsilon}\right\|_{W^{2, p}(\Omega)}^{p} \leq C .
$$

Moreover, there exist $\gamma \in(0,1)$ and $C^{\prime}>0$ such that for any $\varepsilon \in(0,1)$ and all $t>T$ the solution $\left(u_{\varepsilon}, v_{\varepsilon}, w_{\varepsilon}\right)$ of $(3.1)$ satisfies

$$
\left\|u_{\varepsilon}\right\|_{C^{1+\gamma, \frac{\gamma}{2}}(\bar{\Omega} \times[t, t+1])}+\left\|w_{\varepsilon}\right\|_{C^{1+\gamma, \frac{\gamma}{2}}(\bar{\Omega} \times[t, t+1])} \leq C^{\prime} .
$$

Proof: The proof of (11.1) is based on an inductive argument relying on the conditional eventual regularity estimates from Lemma 8.2. We set $q_{(-1)}:=\beta-1$ and for $k \in \mathbb{N}_{0}$ define $q_{(k)}:=k+\beta$. We claim that for all $k \in \mathbb{N}$ we have $q_{(k-1)}>\max \left\{\beta-1, \frac{1}{\beta-2}\right\}$ and that there are $T_{(k)}>0$ and $C_{(k)}>0$ such that for each $\varepsilon \in(0,1)$ and all $t>T_{(k)}$

$$
\int_{t}^{t+1}\left\|u_{\varepsilon}\right\|_{W^{2, q_{(k-2)}(\Omega)}}^{q_{(k-2)}}+\int_{t}^{t+1}\left\|w_{\varepsilon}\right\|_{W^{2, q}(k)(\Omega)}^{q_{(k)}}+\int_{\Omega} v_{\varepsilon}^{1-\beta+q_{(k)}}(\cdot, t)+\int_{t}^{t+1} \int_{\Omega} v_{\varepsilon}^{q_{(k)}} \leq C_{(k)} .
$$

It can easily be checked, that $\beta>1+\sqrt{2}$ implies $q_{(0)}=\beta>\max \left\{\beta-1, \frac{1}{\beta-2}\right\}$. Moreover, noting that $q_{(0)}-\beta+2=2<\beta$, we conclude from Lemma 4.1, Corollary 8.1 and Lemma 10.2 that the conditions of Lemma 8.2 are met for $q=q_{(0)}=\beta$ and that, hence, there are $T_{(1)}>0$ and $C_{(1)}>0$ such that

$$
\begin{aligned}
& \int_{t}^{t+1}\left\|u_{\varepsilon}\right\|_{W^{2, q}(-1)(\Omega)}^{q_{(-1)}}+\int_{t}^{t+1}\left\|w_{\varepsilon}\right\|_{W^{2, q_{(1)}(\Omega)}}^{q_{(1)}}+\int_{\Omega} v_{\varepsilon}^{1-\beta+q_{(1)}}+\int_{t}^{t+1} \int_{\Omega} v_{\varepsilon}^{q_{(1)}} \\
= & \int_{t}^{t+1}\left\|u_{\varepsilon}\right\|_{W^{2, \beta-1}(\Omega)}^{\beta-1}+\int_{t}^{t+1}\left\|w_{\varepsilon}\right\|_{W^{2, \beta+1}(\Omega)}^{\beta+1}+\int_{\Omega} v_{\varepsilon}^{2}+\int_{t}^{t+1} \int_{\Omega} v_{\varepsilon}^{\beta+1} \leq C_{(1)}
\end{aligned}
$$

for each $\varepsilon \in(0,1)$ and all $t>T_{(1)}$, proving that the claim holds for $k=1$. Now, assuming that for an arbitrary but fixed $k \in \mathbb{N}$ we have $q_{(k-1)}>\max \left\{\beta-1, \frac{1}{\beta-2}\right\}$ and $T_{(k)}>0, C_{(k)}>0$ such that (11.3) holds for each $\varepsilon \in(0,1)$ and all $t>T_{(k)}$, we proceed to show the inductive step from $k$ to $k+1$. From the definition of $q_{(k-1)}$ we find that $q_{(k)}=q_{(k-1)}+1>\max \left\{\beta-1, \frac{1}{\beta-2}\right\}$ and by the induction hypothesis there is some $C^{\prime}>0$ such that

$$
\int_{t}^{t+1}\left\|w_{\varepsilon}\right\|_{W^{2, \beta+k}(\Omega)}^{\beta+k}+\int_{t}^{t+1} \int_{\Omega} v_{\varepsilon}^{2+k} \leq \int_{t}^{t+1}\left\|w_{\varepsilon}\right\|_{W^{2, \beta+k}(\Omega)}^{\beta+k}+\int_{t}^{t+1} \int_{\Omega} v_{\varepsilon}^{\beta+k}+C^{\prime} \leq C_{(k)}+C^{\prime}
$$

holds for each $\varepsilon \in(0,1)$ and all $t>T_{(k)}$. Together with Lemma 10.2 these estimates ensure that Lemma 8.2 becomes applicable for $q=q_{(k)}=\beta+k$. Hence, we can conclude that there are $T_{(k+1)}>0$ and $C_{(k+1)}>0$ such that (11.3) remains true when inserting $k+1$, implying that, actually, for any $k \in \mathbb{N}$ we can find $T_{(k)}>0$ and $C_{(k)}>0$ such that $(11.3)$ holds for each $\varepsilon \in(0,1)$ and all $t>T_{(k)}$. This proves the first assertion of the lemma due to $q_{(k)}>k+2$ for all $k \in \mathbb{N}$. From this we can also readily derive (11.2) in view of known embedding results (e.g. [2, Theorem 1.1]) by taking $k$ sufficiently large.

Since the previous lemma contains sufficiently strong information on the derivatives of $u_{\varepsilon}$, we can now employ the maximal Sobolev estimate one final time to the second equation of (3.1) in order to obtain an eventual Hölder estimate for $v_{\varepsilon}$. 


\section{Lemma 11.2.}

Assume $\mu>0, \alpha>1+\sqrt{2}$ and $\beta>1+\sqrt{2}$ and that $r \in C^{1}(\bar{\Omega} \times[0, \infty)) \cap L^{\infty}(\Omega \times(0, \infty)) \cap$ $L^{1}\left((0, \infty) ; L^{\infty}(\Omega)\right)$ fulfills (1.10). Then there exist $\gamma \in(0,1), T>0$ and $C>0$ such that for each $\varepsilon \in(0,1)$ and all $t>T$ the solution $\left(u_{\varepsilon}, v_{\varepsilon}, w_{\varepsilon}\right)$ of $(3.1)$ satisfies

$$
\left\|v_{\varepsilon}\right\|_{C^{1+\gamma, \frac{\gamma}{2}}(\bar{\Omega} \times[t, t+1])} \leq C .
$$

Proof: Given $\theta>1$ we pick $\sigma \in(1, \theta)$ and note that according to Lemma 11.1 we can fix $t_{0}>0$, and $C_{1}>0$ such that for all $t>t_{0}$ and each $\varepsilon \in(0,1)$ we have

$$
\left\|\nabla u_{\varepsilon}\right\|_{L^{\infty}(\Omega \times(t, t+2))}+\int_{t}^{t+2}\left\|\Delta u_{\varepsilon}\right\|_{L^{2 \theta}(\Omega)}^{\theta} \leq C_{1}
$$

and $C_{2}>0$ such that

$$
\left\|v_{\varepsilon}\right\|_{L^{\infty}\left(\left(t_{0}, \infty\right) ; L^{2 \theta}(\Omega)\right)}+\left\|v_{\varepsilon}\right\|_{L^{\infty}\left(\left(t_{0}, \infty\right) ; L^{\beta \theta}(\Omega)\right)}+\left\|v_{\varepsilon}\right\|_{L^{\infty}\left(\left(t_{0}, \infty\right) ; L^{\theta}(\Omega)\right)}+\left\|v_{\varepsilon}\right\|_{L^{\infty}\left(\left(t_{0}, \infty\right) ; L^{\sigma}(\Omega)\right)} \leq C_{2}
$$

is valid for each $\varepsilon \in(0,1)$. Now, in order to prepare maximal Sobolev regularity arguments in a fashion similar to Lemma 8.2, we let $\xi:=\xi_{t_{0}}$ again be given by Definition 4.2 and observe that $\xi v_{\varepsilon}$ satisfies

$$
\left(\xi v_{\varepsilon}\right)_{t}=\Delta\left(\xi v_{\varepsilon}\right)-\nabla\left(\xi v_{\varepsilon}\right) \nabla u_{\varepsilon}-\xi v_{\varepsilon} \Delta u_{\varepsilon}+\xi g\left(v_{\varepsilon}\right)+\xi^{\prime} v_{\varepsilon} \quad \text { in } \Omega \times\left(t_{0}, \infty\right)
$$

with $\nabla\left(\xi v_{\varepsilon}\right) \cdot \nu=0$ on $\partial \Omega \times\left(t_{0}, \infty\right)$ and $\xi v_{\varepsilon}\left(\cdot, t_{0}\right)=0$ in $\Omega$. Hence, maximal Sobolev regularity estimates for the Neumann heat semigroup $([13,11])$ entail the existence of $C_{3}>0$ such that for each $\varepsilon \in(0,1)$ we may estimate

$$
\begin{array}{r}
\int_{t_{0}}^{t_{0}+2}\left\|\left(\xi v_{\varepsilon}\right)_{t}\right\|_{L^{\theta}(\Omega)}^{\theta}+\int_{t_{0}}^{t_{0}+2}\left\|\xi v_{\varepsilon}\right\|_{W^{2, \theta}(\Omega)}^{\theta} \leq C_{3} \int_{t_{0}}^{t_{0}+2}\left\|\nabla\left(\xi v_{\varepsilon}\right) \cdot \nabla u_{\varepsilon}\right\|_{L^{\theta}(\Omega)}^{\theta}+C_{3} \int_{t_{0}}^{t_{0}+2}\left\|\xi v_{\varepsilon} \Delta u_{\varepsilon}\right\|_{L^{\theta}(\Omega)}^{\theta} \\
+C_{3} \int_{t_{0}}^{t_{0}+2}\left\|\xi g\left(v_{\varepsilon}\right)\right\|_{L^{\theta}(\Omega)}^{\theta}+C_{3} \int_{t_{0}}^{t_{0}+2}\left\|\xi^{\prime} v_{\varepsilon}\right\|_{L^{\theta}(\Omega)}^{\theta}
\end{array}
$$

Making use of Hölder's inequality, (1.6) and the fact that $\xi \leq 1$ on $\mathbb{R}$, we find $C_{4}>0$ such that for each $\varepsilon \in(0,1)$

$$
\begin{aligned}
& \int_{t_{0}}^{t_{0}+2}\left\|\xi v_{\varepsilon}\right\|_{W^{2, \theta}(\Omega)}^{\theta} \\
\leq & C_{3}\left\|\nabla u_{\varepsilon}\right\|_{L^{\infty}\left(\left(\Omega \times\left(t_{0}, t_{0}+2\right)\right)\right.} \int_{t_{0}}^{t_{0}+2} \int_{\Omega}\left|\nabla\left(\xi v_{\varepsilon}\right)\right|^{\theta}+C_{3} \int_{t_{0}}^{t_{0}+2}\left\|v_{\varepsilon}\right\|_{L^{2 \theta}(\Omega)}^{\theta}\left\|\Delta u_{\varepsilon}\right\|_{L^{2 \theta}(\Omega)}^{\theta} \\
& +C_{4} \int_{t_{0}}^{t_{0}+2} \int_{\Omega} v_{\varepsilon}^{\beta \theta}+C_{4}+C_{3}\left\|\xi^{\prime}\right\|_{L^{\infty}(\mathbb{R})}^{\theta} \int_{t_{0}}^{t_{0}+2} \int_{\Omega} v_{\varepsilon}^{\theta} .
\end{aligned}
$$

Since a combination of the Gagliardo-Nirenberg inequality and Young's inequality provides $C_{5}, C_{6}>0$ and $a=\frac{\frac{1}{2}+\frac{1}{\sigma}-\frac{1}{\theta}}{1+\frac{1}{\sigma}-\frac{1}{\theta}}<1$ such that

$$
C_{1} C_{3}\|\phi\|_{W^{1, \theta}(\Omega)}^{\theta} \leq C_{5}\|\phi\|_{W^{2, \theta}(\Omega)}^{a \theta}\|\phi\|_{L^{\sigma}(\Omega)}^{(1-a) \theta} \leq \frac{1}{2}\|\phi\|_{W^{2, \theta}(\Omega)}^{\theta}+C_{6}\|\phi\|_{L^{\sigma}(\Omega)}^{\theta} \quad \text { for all } \phi \in W^{2, \theta}(\Omega),
$$

we conclude from $(11.4),(11.5)$ and (11.6) that for each $\varepsilon \in(0,1)$ we have

$$
\frac{1}{2} \int_{t_{0}}^{t_{0}+2}\left\|\xi v_{\varepsilon}\right\|_{W^{2, \theta}(\Omega)}^{\theta} \leq 2 C_{2}^{\theta} C_{6}+C_{1} C_{2}^{\theta} C_{3}+2 C_{2}^{\beta \theta} C_{4}+C_{4}+2 C_{2}^{\theta} C_{3}\left\|\xi^{\prime}\right\|_{L^{\infty}(\mathbb{R})}^{\theta} .
$$

Hence, noting that $\xi \equiv 1$ on $\left(t_{0}+1, t_{0}+2\right)$, we can for any $\theta>1$ find some $C_{7}>0$ such that for each $\varepsilon \in(0,1)$ and all $t>T:=t_{0}+1$ we have

$$
\int_{t}^{t+1}\left\|v_{\varepsilon}\right\|_{W^{2, \theta}(\Omega)}^{\theta} \leq C_{7}
$$

The asserted Hölder regularity now follows from an application of the known embedding results e.g. presented in [2, Theorem 1.1]. 
With the prepared regularity information we can now rely on the Arzelà-Ascoli theorem and parabolic regularity theory to verify that the global generalized solution obtained in Theorem 1.1 actually solves (1.5) classically at least after some smoothing time $T_{\diamond}>0$. Reasoning along these lines has previously been utilized in similar settings and can e.g. be found in [41, Lemma 7.13], [20, Lemma 3.12] and [6, Lemma 7.5].

Proof of Theorem 1.3: Making use of Lemma 11.1 and Lemma 11.2 we can pick some $\gamma^{\prime} \in(0,1)$, $T>0$ and $C_{1}>0$ such that for each $\varepsilon \in(0,1)$ and any $t>T$

$$
\left\|u_{\varepsilon}\right\|_{C^{1+\gamma^{\prime}, \frac{\gamma^{\prime}}{2}}(\bar{\Omega} \times[t, t+1])}+\left\|v_{\varepsilon}\right\|_{C^{1+\gamma^{\prime}, \frac{\gamma^{\prime}}{2}}(\bar{\Omega} \times[t, t+1])}+\left\|w_{\varepsilon}\right\|_{C^{1+\gamma^{\prime}, \frac{\gamma^{\prime}}{2}}(\bar{\Omega} \times[t, t+1])} \leq C_{1} .
$$

Denoting by $\left(\varepsilon_{j}\right)_{j \in \mathbb{N}}$ the sequence obtained in Proposition 6.1 and with $(u, v, w)$ the corresponding limit object, we can draw on the Arzelà-Ascoli theorem to find that for some $\gamma_{0} \in\left(0, \gamma^{\prime}\right)$

$$
u_{\varepsilon} \rightarrow u, \quad v_{\varepsilon} \rightarrow v, \quad w_{\varepsilon} \rightarrow w \quad \text { in } C^{1+\gamma_{0}, \frac{\gamma_{0}}{2}}(\bar{\Omega} \times[t, t+1])
$$

along a subsequence of $\left(\varepsilon_{j}\right)_{j \in \mathbb{N}}$. In particular, $u$ and $v$ are bounded on $\bar{\Omega} \times[t, t+1]$, which in light of $f, g \in C^{1}([0, \infty))$, implies that

$$
f(u), g(v) \in C^{\gamma^{\prime}, \frac{\gamma_{0}}{2}}(\bar{\Omega} \times[t, t+1]) .
$$

Letting $\xi_{1}:=\xi_{T}$ be provided by Definition 4.2 we find that $W=\xi_{1} w$ solves the problem

$$
W_{t}=\Delta W+H, \quad W(T)=0,\left.\quad \frac{\partial W}{\partial \nu}\right|_{\partial \Omega}=0
$$

with $H=-(u+v) \xi_{1} w-\mu \xi_{1} w+r \xi_{1}+\xi_{1}^{\prime} w \in C^{\gamma_{0}}, \frac{\gamma_{0}}{2}(\bar{\Omega} \times(T, \infty))$. Hence, parabolic theory ([18, III.5.1 and IV.5.3]) tells us that there is some $\gamma_{1} \in\left(0, \gamma_{0}\right)$ such that

$$
w \in C^{2+\gamma_{1}, 1+\frac{\gamma_{1}}{2}}(\bar{\Omega} \times[T+1, \infty)) .
$$

Similarly, setting $\xi_{2}:=\xi_{T+1}$ we notice that $U=\xi_{2} u$ is a solution of the problem

$$
U_{t}=\Delta U-A \cdot \nabla U+B, \quad U(T+1)=0,\left.\quad \frac{\partial U}{\partial \nu}\right|_{\partial \Omega}=0
$$

where $A=\nabla w \in C^{\gamma_{1}, \frac{\gamma_{1}}{2}}(\bar{\Omega} \times(T+1, \infty))$ and $B=-\xi_{2} u \Delta w+\xi_{2} f(u)+\xi_{2}^{\prime} u \in C^{\gamma_{1}, \frac{\gamma_{1}}{2}}(\bar{\Omega} \times(T+1, \infty))$ again imply by parabolic theory $\left(\left[18\right.\right.$, III.5.1 and IV.5.3]) that there is $\gamma_{2} \in\left(0, \gamma_{1}\right)$ such that

$$
u \in C^{2+\gamma_{2}, 1+\frac{\gamma_{2}}{2}}(\bar{\Omega} \times[T+2, \infty)) .
$$

Repeating the same arguments a third time with $\xi_{3}:=\xi_{T+2}$ for the solution $V=\xi_{3} v$ of

$$
V_{t}=\Delta V-A^{\prime} \cdot \nabla V+B^{\prime}, \quad V(T+2)=0,\left.\quad \frac{\partial V}{\partial \nu}\right|_{\partial \Omega}=0,
$$

with $A^{\prime}=\nabla u \in C^{\gamma_{2}, \frac{\gamma_{2}}{2}}(\bar{\Omega} \times(T+3, \infty))$ and $B^{\prime}=-\xi_{3} v \Delta u+\xi_{3} g(v)+\xi_{3}^{\prime} v \in C^{\gamma_{2}, \frac{\gamma_{2}}{2}}(\bar{\Omega} \times(T+2, \infty))$ lastly provides $\gamma_{3} \in\left(0, \gamma_{2}\right)$ such that

$$
v \in C^{2+\gamma_{3}, 1+\frac{\gamma_{3}}{2}}(\bar{\Omega} \times[T+3, \infty)) .
$$

As a consequence of $(11.7),(11.8),(11.9)$ we readily obtain the regularity assertions of the theorem, which, upon letting $T_{\diamond}:=T+3$, imply that $(u, v, w)$ solves $(1.5)$ classically in $\Omega \times\left(T_{\diamond}, \infty\right)$.

\section{Acknowledgements}

The author acknowledges support of the Deutsche Forschungsgemeinschaft in the context of the project Emergence of structures and advantages in cross-diffusion systems (project no. 411007140). 


\section{References}

[1] H. Amann. Nonhomogeneous linear and quasilinear elliptic and parabolic boundary value problems. In Function spaces, differential operators and nonlinear analysis, Volume 133 of Teubner-Texte Math., pp. 9-126. Teubner, Stuttgart, 1993.

[2] H. Amann. Compact embeddings of vector-valued Sobolev and Besov spaces. Glas. Mat. Ser. III, 35(55)(1):161-177, 2000 .

[3] N. Bellomo and J. Soler. On the mathematical theory of the dynamics of swarms viewed as complex systems. Math. Mod. Meth. Appl. Sci., 22(supp01):1140006, 2012.

[4] N. Bellomo, A. Bellouquid, Y. Tao, and M. Winkler. Toward a mathematical theory of Keller-Segel models of pattern formation in biological tissues. Math. Mod. Meth. Appl. Sci., 25(9):1663-1763, 2015.

[5] T. Black. Global solvability of chemotaxis-fluid systems with nonlinear diffusion and matrix-valued sensitivities in three dimensions. Nonlinear Anal., 180:129-153, 2019.

[6] T. Black. The Stokes limit in a three-dimensional chemotaxis-Navier-Stokes system. 2019. arXiv:1902.06237 - To appear in J. Math. Fluid Mech.

[7] R. Eftimie, G. de Vries, and M. A. Lewis. Complex spatial group patterns result from different animal communication mechanisms. Proc. Natl. Acad. Sci. U.S.A., 104(17):6974-6979, 2007.

[8] R. A. Fisher. The wave of advance of advantageous genes. Annals of Eugenics, 7(4):355-369, 1937.

[9] A. Friedman. Partial differential equations. Holt, Rinehart and Winston, Inc., New York-Montreal, Que.-London, 1969.

[10] G. Furioli, A. Pulvirenti, E. Terraneo, and G. Toscani. Fokker-Planck equations in the modeling of socio-economic phenomena. Math. Mod. Meth. Appl. Sci., 27(01):115-158, Jan. 2017.

[11] Y. Giga and H. Sohr. Abstract $L^{p}$ estimates for the Cauchy problem with applications to the Navier-Stokes equations in exterior domains. J. Funct. Anal., 102(1):72-94, 1991.

[12] M. A. Herrero and J. J. L. Velázquez. A blow-up mechanism for a chemotaxis model. Ann. Sc. Norm. Super. Pisa Cl. Sci, 24:633-683, 1997.

[13] M. Hieber and J. Prüss. Heat kernels and maximal $L^{p}-L^{q}$ estimates for parabolic evolution equations. Comm. Part. Diff. Eq., 22(9-10):1647-1669, 1997.

[14] T. Hillen and K. J. Painter. A user's guide to PDE models for chemotaxis. J. Math. Biol., 58(1-2):183-217, 2009.

[15] W. Hoffman, D. Heinemann, and J. A. Wiens. The ecology of seabird feeding flocks in Alaska. Auk, 98(3):437-456, 1981.

[16] D. Horstmann and G. Wang. Blow-up in a chemotaxis model without symmetry assumptions. Eur. J. Appl. Math., 12(2):159-177, 2001.

[17] A. Kolmogorov, I. Petrovskii, and N. Piskunov. A study of the diffusion equation with increase in the amount of substance, and its application to a biological problem. In V. M. Tikhomirov, editor, Selected Works of A.N. Kolmogorov: Volume I: Mathematics and Mechanics, pp. 242-270. Springer Netherlands, Dodrecht, 1991.

[18] O. A. Ladyženskaja, V. A. Solonnikov, and N. N. Ural'ceva. Linear and quasilinear equations of parabolic type. Translations of mathematical monographs. American Mathematical Society, 1968.

[19] E. Lankeit and J. Lankeit. Classical solutions to a logistic chemotaxis model with singular sensitivity and signal absorption. Nonlinear Anal. Real World Appl., 46:421-445, 2019.

[20] J. Lankeit. Long-term behaviour in a chemotaxis-fluid system with logistic source. Math. Mod. Meth. Appl. Sci., 26 (11):2071-2109, 2016

[21] J. Lankeit and Y. Wang. Global existence, boundedness and stabilization in a high-dimensional chemotaxis system with consumption. Discrete Contin. Dyn. Syst., 37(12):6099-6121, 2017.

[22] J. Lankeit and M. Winkler. A generalized solution concept for the Keller-Segel system with logarithmic sensitivity: global solvability for large nonradial data. NoDEA Nonlinear Differential Equations Appl., 24(4):Art. 49, 33, 2017.

[23] J. Lankeit and M. Winkler. Facing low regularity in chemotaxis systems. Jahresber. Dtsch. Math.-Ver., 2019.

[24] J. M. Lee, T. Hillen, and M. A. Lewis. Pattern formation in prey-taxis systems. Journal of Biological Dynamics, 3 (6):551-573, 2009 .

[25] X. Li. Global existence and uniform boundedness of smooth solutions to a parabolic-parabolic chemotaxis system with nonlinear diffusion. Bound. Value Probl., 2015(1):1-17, 2015.

[26] Y. Liu. Global existence and boundedness of classical solutions to a forager-exploiter model with volume-filling effects. Nonlinear Anal. Real World Appl., 50:519-531, 2019.

[27] N. Mizoguchi and M. Winkler. Blow-up in the two-dimensional parabolic Keller-Segel system. 2013. Preprint.

[28] M. B. Short, M. R. D’Orsogna, V. B. Pasour, G. E. Tita, P. J. Brantingham, A. L. Bertozzi, and L. B. Chayes. A statistical model of criminal behavior. Math. Mod. Meth. Appl. Sci., 18(supp01):1249-1267, 2008.

[29] J. Simon. Compact sets in the space $L^{p}(0, T ; B)$. Ann. Mat. Pura Appl. (4), 146:65-96, 1987.

[30] N. Tania, B. Vanderlei, J. P. Heath, and L. Edelstein-Keshet. Role of social interactions in dynamic patterns of resource patches and forager aggregation. Proc. Natl. Acad. Sci. U.S.A., 109(28):11228-11233, 2012.

[31] Y. Tao. Boundedness in a chemotaxis model with oxygen consumption by bacteria. J. Math. Anal. Appl., 381(2): $521-529,2011$

[32] Y. Tao and M. Winkler. Eventual smoothness and stabilization of large-data solutions in a three-dimensional chemotaxis system with consumption of chemoattractant. J. Differential Equations, 252(3):2520 - 2543, 2012. 
[33] Y. Tao and M. Winkler. Large time behavior in a forager-exploiter model with different taxis strategies for two groups in search of food. Math. Mod. Meth. Appl. Sci., pp. 1-32, 2019.

[34] J. Toner and Y. Tu. Flocks, herds, and schools: A quantitative theory of flocking. Phys. Rev. E, 58(4):4828-4858, 1998.

[35] M. Winkler. Boundedness in the higher-dimensional parabolic-parabolic chemotaxis system with logistic source. Comm. Part. Diff. Eq., 35:1516-1537, 2010.

[36] M. Winkler. Aggregation vs. global diffusive behavior in the higher-dimensional Keller-Segel model. J. Differential Equations, 248(12):2889-2905, 2010.

[37] M. Winkler. Global large-data solutions in a chemotaxis-(Navier-)Stokes system modeling cellular swimming in fluid drops. Comm. Part. Diff. Eq., 37(2):319-351, 2012.

[38] M. Winkler. Finite-time blow-up in the higher-dimensional parabolic-parabolic Keller-Segel system. J. Math. Pures Appl., 100(5):748 - 767, 2013.

[39] M. Winkler. Stabilization in a two-dimensional chemotaxis-Navier-Stokes system. Arch. Ration. Mech. Anal., 211 (2):455-487, 2014

[40] M. Winkler. Large-data global generalized solutions in a chemotaxis system with tensor-valued sensitivities. SIAM J. Math. Anal., 47(4):3092-3115, 2015.

[41] M. Winkler. How far do chemotaxis-driven forces influence regularity in the Navier-Stokes system? Trans. Amer. Math. Soc., 369(5):3067-3125, 2017.

[42] M. Winkler. Global generalized solutions to a multi-dimensional doubly tactic resource consumption model accounting for social interactions. Math. Mod. Meth. Appl. Sci., 29(3):373-418, 2019.

[43] S. Wu, J. Shi, and B. Wu. Global existence of solutions and uniform persistence of a diffusive predator-prey model with prey-taxis. J. Differential Equations, 260(7):5847-5874, 2016. 